\title{
Parametric Analysis of Excited Round Jets - Numerical Study
}

\author{
Artur Tyliszczak • Bernard J. Geurts
}

Received: 24 October 2013 / Accepted: 2 May 2014 / Published online: 29 May 2014

(C) Springer Science+Business Media Dordrecht 2014

\begin{abstract}
A computational analysis of excited round jets is presented with emphasis on jet bifurcation phenomenon due to superposition of axial and flapping forcing terms. Various excitation parameters are examined including the amplitudes of the forcing, their frequencies and phase shift. It is shown that alteration of these parameters significantly influences the spatial jet evolution. This dependence may be used to control the jet behaviour in a wide range of qualitatively different flow structures, starting from a modification of the spreading rate of a single connected jet, through large scale deformation of an asymmetric jet, onto jet bifurcation leading to a doubly and even triply split time-averaged jet, displaying different strengths and locations of the branches. We establish that: (i) jet splitting is possible only when the amplitudes of the forcing terms are comparable to or larger than the level of natural turbulence; (ii) the angle between the developing jet branches can be directly controlled by the frequency of the axial forcing and the phase shift between axial and flapping forcing. An optimum forcing frequency is determined, leading to the largest spreading rate.
\end{abstract}

Keywords Flow control · Jet bifurcation · Axial and flapping forcing · Spreading rate

\section{Introduction}

Flow control plays a very important role in various technological processes in which a suitable alteration of the flow field would lead to considerable improvement of efficiency, safety or performance of a fluid-mechanical operation step. Flow control may be divided into two categories, a passive and an active control with the latter distinguishing

\footnotetext{
A. Tyliszczak $(\bowtie)$

Czestochowa University of Technology Al. Armii Krajowej 21, 42-200 Czestochowa, Poland

e-mail: atyl@imc.pcz.czest.pl
}

\section{B. J. Geurts}

Multiscale Modelling and Simulation University of Twente P.O. Box 217, 7500 AE Enschede, The Netherlands e-mail: B.J.Geurts@utwente.nl 
predetermined and interactive methods [1]. Passive flow control involves manipulation of the flow field without adding any external energy. It is often a result of trial-and-error procedures based on geometric shaping or adding fixed elements (obstacles, grooves, swirlers). Active methods of control involve energy input whose type and level may be fixed (predetermined approach) or may be varying depending on the instantaneous flow behaviour (interactive approach). In this paper we concentrate on predetermined flow control methods and its ability to alter the qualitative structure of a circular jet flow. In the 70s Crow an Champagne [2] showed that with properly chosen excitation the jet may exhibit qualitatively different behaviour compared to the natural unexcited jet. They managed to intensify turbulence intensity and mixing. This was among the first reported results on active jet control triggering important research both experimentally and numerically. We contribute to this presenting new regimes of jet manipulation and jet splitting as a result of properly selected upstream perturbations. In particular, we show that high-resolution large-eddy simulation can be used [3-5] to accurately understand dynamics of turbulent jets and adopt this approach to perform a comprehensive parameter study, investigating jet response such as spreading rate and jet splitting.

A very interesting phenomenon occurring under suitable excitation conditions is jet bifurcation which implies a jet to split into two separate well defined streams (branches) with vortex rings moving along its branches. It was shown experimentally [6-8] that this bifurcation phenomenon can be generated by applying a superposition of an axial and a flapping forcing at the jet nozzle, with properly chosen frequencies, $f_{a}$ and $f_{h}$, respectively. The necessary conditions leading to bifurcation were determined in terms of the axial forcing frequency and the ratio, $f_{a} / f_{h}$. It was shown that bifurcation appears when $f_{a} / f_{h}=2$ and is most pronounced when $f_{a}$ is close to the so-called preferred mode frequency, $f_{p}$, i.e., the frequency at which an axisymmetric disturbance in the initial jet region is amplified and attains the maximum growth [2, 9]. It was shown in [7] that at Reynolds numbers $10^{4}<\operatorname{Re}<10^{5}$ bifurcation characteristics are weakly dependent on Re. Numerical simulations performed with Direct Numerical Simulation (DNS) [10-13] and Large Eddy Simulation (LES) [1416] reproduced and confirmed these experimental results. Moreover, new findings were revealed, among others, for low Reynolds number jets $(\operatorname{Re}=1500)$ the flapping excitation is sufficient to obtain jet bifurcation and there is no need to also include the axial excitation [12] as was later confirmed in [15]. Such behaviour was attributed to the existence of strong primary vortex rings which at low Reynolds number are not distorted by natural turbulence or flapping forcing. In contrast, at higher Reynolds numbers the flapping excitation rapidly distorts these vortices and therefore axial forcing is included to amplify the vortices and retain the two-fold split jet $[12,15]$. The effect of the forcing frequency was studied in [15] with respect to alteration of the flapping frequency $f_{h}$ only, while $f_{a}$ was kept equal to $f_{p}$. A different setting of the forcing frequencies was studied in [13] where a combination of the axial and flapping forcing was used as an optimisation parameter. A low amplitude forcing was applied to improve the mixing and spreading rate of the jet. The most efficient combination was obtained with $f_{a}$ slightly bigger than $2 f_{p}$ and with $f_{h}$ equal to $f_{p}$.

The present study extends the numerical research and focusses on the effect of alteration of the amplitude, frequency and phase shift of the forcing. It is shown that these parameters significantly changes the flow behaviour and can be used to increase spreading rate of a jet or to create an asymmetric jet or the bifurcating jets with splitting occurring at different position and with different angle between the jet branches.

The paper is organised as follow: Section 2 is devoted to a description of the numerical method used in the computation; it also presents details of the computational setup such as domain, boundary conditions and computational meshes. Section 3 is devoted to a validation 
of the code based on a natural non-excited jet study for which we compare our results with findings in literature; Section 4 deals with excited jets and shows that the applied numerical algorithm can capture the jet bifurcation very accurately. Conclusions and future research directions are formulated in Section 5.

\section{Computational Approach to Forced Turbulent Jets}

In this section we first briefly sketch the main characteristics of the flow solver used for this study (Subsection 2.1) and subsequently describe the computational setup in terms of domain and boundary conditions (Subsection 2.2).

\subsection{LES solver}

The flow solver used in this work is an academic high-order code based on the low Mach number approximation $[17,18]$. This code (SAILOR) may be used for solving a wide range of flows under various conditions, varying from isothermal and constant density to situations with considerable density and temperature variations. For research purposes the SAILOR code includes a variety of sub-grid models used when the code is operated in Large Eddy simulation (LES) mode [19, 20]. In the present work we consider constant density flow and incorporate the sub-grid model as proposed by Vreman [21]. In this model the subgrid viscosity vanishes in laminar flows or pure shear regions. This is an important aspect in jet flows with low turbulent intensity at the inlet conditions. An excess of dissipation coming from the subgrid part would hinder the transition and developed turbulence regimes - this is not the case with the selected model.

The SAILOR code was used previously in various studies including laminar/turbulent transition in near-wall flows [22], free jet flows [23, 24], multi-phase flows [25] and flames $[26,27]$. The SAILOR code is based on a projection method with time integration performed by a predictor-corrector (Adams-Bashforth / Adams-Moulton) method [28]. The spatial discretization is based on 6th order compact differencing developed for half-staggered meshes [29]. Unlike in the fully staggered approach the velocity nodes are common for all three velocity components whereas the pressure nodes are moved half a grid size from the velocity nodes. This greatly facilitates implementation of the code and is computationally efficient as there is only a small amount of interpolation between the nodes. As shown in [29] the staggering of the pressure nodes is sufficient to ensure a strong velocity-pressure coupling which eliminates the well known pressure oscillations occurring on collocated meshes. Additionally, the shifting of the pressure nodes into the centres on the computational cells simplifies implementation of the boundary conditions as there is no need to specify the pressure on the boundaries.

\subsection{Computational domain and boundary conditions}

A schematic view of the flow set-up showing the jet nozzle together with a downstream region is presented in Fig. 1. In the present work we do not consider the inner geometry of the nozzle. Hence, the domain is a simple rectangular box which starts in the plane of the nozzle exit. The inlet boundary conditions characterize the effect of the nozzle and are specified in terms of the mean velocity profile to which fluctuating components are superimposed. Outside the jet region a co-flow with low velocity $U_{c}$ is added to mimic the natural suction and entrainment from the surroundings. At the side boundaries the streamwise 
Fig. 1 Schematic view of the analyzed configuration. The computational domain is a box between inlet and outlet planes

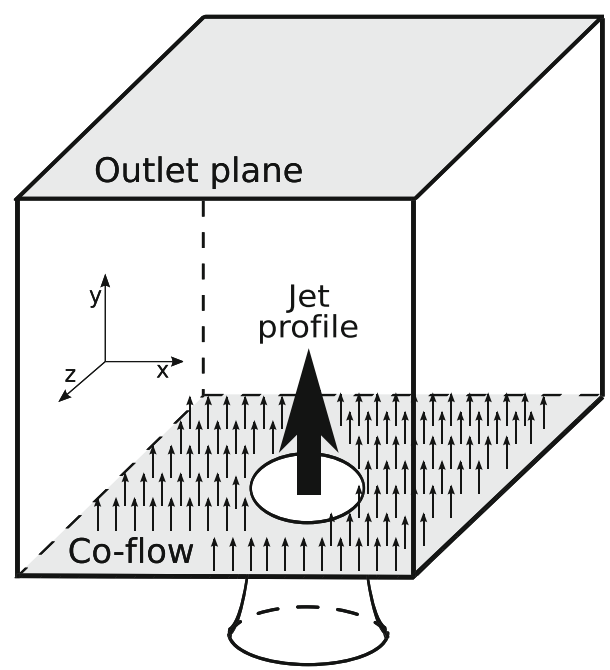

velocity is taken equal to the co-flow velocity $U_{c}$ while the velocity components normal to the boundary of the domain are set equal to zero. At the outlet plane all velocity components are computed from the convective boundary condition $\partial u_{i} / \partial t+C \partial u_{i} / \partial y=0$ with $C$ being the convection velocity which is computed as the $(x, z)$ averaged mean velocity in the outlet plane, at every time step. This boundary condition is found to be stable and allows the flow structures to leave the domain with only small distortion.

Experimental data [8] show that the splitting of the mean jet can start relatively closely to the nozzle, i.e., at 4 to 5 diameters $(D)$ downstream. Because in the present work we are mostly interested in forcing parameters leading to bifurcation and not so much in the far field flow behaviour, the basic computational domain is set to $12 D \times 16 D \times 12 D$ which is large enough to capture the main flow features of relevance to the jet splitting, which are found to be concentrated to a region of size $8 D \times 12 D \times 8 D$ as will be shown later. In order to check a possible influence of the lateral boundaries some additional test computations for selected cases are performed using the domain $16 D \times 16 D \times 16 D$.

\subsubsection{Inlet excitation method}

In experimental research the excitation at the nozzle exit is usually produced by a membrane or loudspeaker located upstream of the nozzle or by obstacles located near the nozzle exit or through mechanical forcing obtained by specially designed actuators placed at the nozzle lip [7, 30, 31]. In the present study we add the excitation as a component of the velocity prescribed at the inlet. Similarly as in $[12,15,16]$ the inlet velocity profile used in the present work is defined as:

$$
u(\vec{x}, t)=u_{\text {mean }}(\vec{x})+u_{\text {excit }}(\vec{x}, t)+u_{\text {turb }}(\vec{x}, t)
$$

where the mean streamwise velocity is taken as the hyperbolic-tangent profile:

$$
u_{\text {mean }}(\vec{x})=\frac{U_{j}+U_{c}}{2}-\frac{U_{j}-U_{c}}{2} \tanh \left(\frac{1}{4} \frac{R}{\delta_{\theta}}\left(\frac{r}{R}-\frac{R}{r}\right)\right)
$$

The mean velocity in the other two directions is taken to be zero. In (2) $U_{j}$ denotes the jet centerline velocity, $U_{c}$ is recalled to denote the co-flow velocity equal to $0.05 U_{j}, r$ measures 
the distance from the centerline, $R$ is the nozzle radius, and $\delta_{\theta}$ is the momentum thickness of the initial shear layer. In all the cases presented in this paper the momentum thickness of the jet is the same as used in [15] and taken as $\delta_{\theta}=0.05 R$ which yields the shear layer thickness $\delta_{99}=0.48 R$ defined here as the region where $U_{c}<u_{\text {mean }}(\vec{x}) \leq 0.99 U_{j}$. The forcing component $u_{\text {excit }}(\vec{x}, t)$ is added to the streamwise velocity only and it is defined as:

$$
u_{\text {excit }}(\vec{x}, t)=\underbrace{A_{a} \sin \left(2 \pi S t_{a} \frac{U_{j}}{D} t\right)}_{\text {Axial forcing }}+\underbrace{A_{h} \sin \left(2 \pi S t_{h} \frac{U_{j}}{D} t+\phi\right) \sin \left(\frac{\pi x}{D}\right)}_{\text {Flapping forcing }}
$$

which is the superposition of axial and flapping forcing shown schematically in Fig. 2. The forcing amplitudes are $A_{a}$ and $A_{h}$ while the Strouhal numbers are defined as $S t_{a}=f_{a} D / U_{j}$ and $S t_{h}=f_{h} D / U_{j}$. Both forcing terms are limited to the jet region $-0.5 \leq r / D \leq 0.5$ and are additionally multiplied by $\tanh \left(\frac{1}{4} \frac{R}{\delta_{\theta}}\left(\frac{r}{R}-\frac{R}{r}\right)\right)$ that provides a smooth transition with the co-flow region. Sample instantaneous forcing profiles are presented in Fig. 3. Note that the flapping forcing is antisymmetric with respect to $x / D=0$ and its maxima are close to the shear layer region. The last term in $(1), u_{t u r b}(\vec{x}, t)$, represents turbulent fluctuations uniformly distributed across the whole jet section. They were computed applying a digital filtering method proposed by Klein et al. [32]. This method guarantees properly correlated velocity fields which reflect realistic turbulent flow conditions.

In computations we analyse the effect of the magnitude of the excitation amplitudes and the turbulence intensity corresponding to $u_{\text {turb }}(\vec{x}, t)$ and computed as $T i=$ $\sqrt{\left\langle u_{\text {turb }}(\vec{x}, t)^{2}\right\rangle}$. Both the excitation amplitudes and the turbulence intensity levels $T i$ will be defined relative to the jet centerline velocity. We will consider cases with low $\left(T i=0.01 U_{j}\right)$ and moderate $\left(T i=0.05 U_{j}\right)$ turbulence levels and excitation amplitudes equal to $A_{a} / U_{j}=A_{h} / U_{j}=0.01,0.05,0.15$.

\subsubsection{Computational parameters}

Most of the computations were performed on a mesh with $128 \times 160 \times 128$ nodes which will be further referred to as the reference grid and denoted as mesh A. In order to determine the dependence of the results on the mesh density some computations have been performed also on two denser meshes with $256 \times 320 \times 256$ nodes (mesh B) and $384 \times 320 \times 384$ nodes (mesh C). Figure 3 shows locations of the grid nodes within the shear layer. Note that for better visibility the profiles in this figure are artificially shifted by $\Delta u=0.2$ and $\Delta u=0.4$.

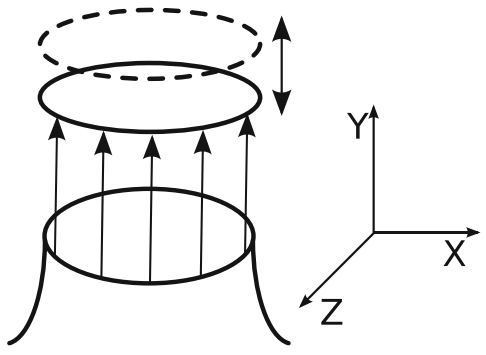

(a) axial excitation

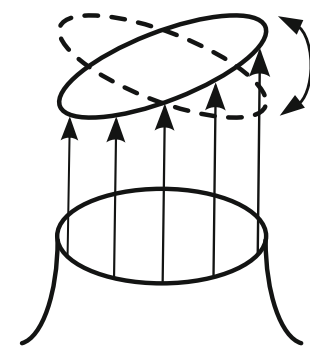

(b) flapping excitation

Fig. 2 Schematic view of the analyzed excitations introduced to excite flow structures in the jet 


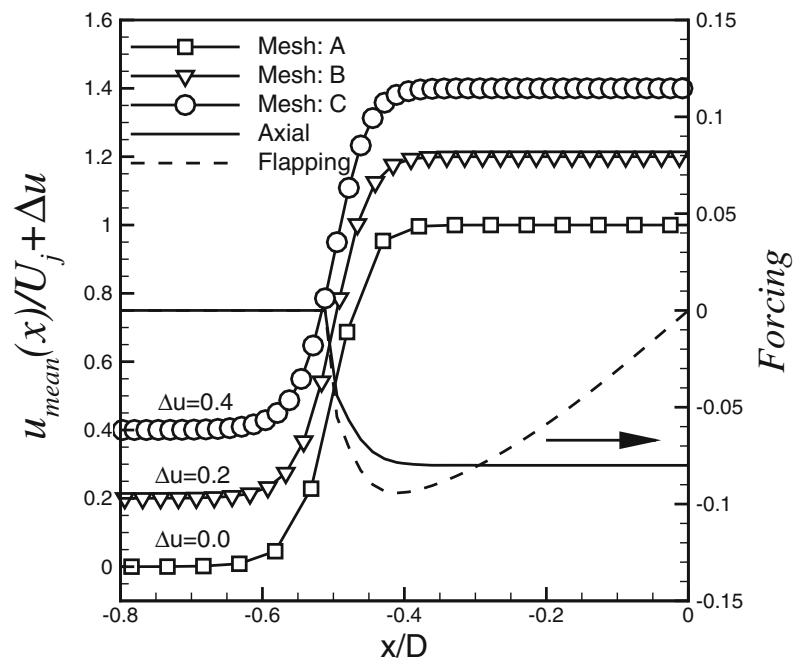

Fig. 3 Lines without symbols denote the profiles of the imposed axial and flapping forcing. Lines with symbols denote the mean axial velocity profiles on different meshes shifted by $\Delta u$ for better visibility - A $(128 \times 160 \times 128)$ with $\Delta u=0 ; \mathrm{B}(256 \times 320 \times 256)$ with $\Delta u=0.2 ; \mathrm{C}(384 \times 320 \times 384)$ with $\Delta u=0.4$

The meshes were stretched axially and radially towards the inlet where the cell sizes were almost uniform. Depending on the applied mesh the initial shear layer of the jet profile was resolved by 5 (mesh A), 9 (mesh B) and 14 (mesh C) nodes. The parameters of all these meshes are given in Table 1. Note that in the case of a wider domain (meshes denoted as $\mathrm{B}(\mathrm{W})$ and $\mathrm{C}(\mathrm{W}))$ the cell sizes are smaller than on the meshes $\mathrm{B}$ and $\mathrm{C}$. This arises from a particular setting of the stretching parameters and was done on purpose to better check the influence of the mesh resolution in the shear layer. The numerical time step was computed dynamically based on the Courant-Friedrichs-Lewy (CFL) criterion with CFL number equal to 0.5 . In all cases the initial conditions corresponded to a uniform velocity equal to the coflowing velocity. The jet was injected impulsively and then tracked as it developed in the domain. After a time $100 \mathrm{D} / U_{j}$ the flow was found to have become virtually independent of the initial condition and the time-averaging procedure started for the next $300 D / U_{j}$. This corresponds to more than 18 flow passes through the flow domain (assuming the jet velocity as the reference velocity).

Table 1 Parameters of the computational meshes. Symbol (W) denotes the wider computational domain

\begin{tabular}{lllll}
\hline Mesh & $N_{x} \times N_{y} \times N_{z}$ & $L_{x} \times L_{y} \times L_{z}$ & $\Delta x=\Delta z$ & $\Delta y$ \\
\hline A & $128 \times 160 \times 128$ & $12 D \times 16 D \times 12 D$ & $0.050 D\left(\approx 0.208 \delta_{99}\right)$ & $0.067 D$ \\
B & $256 \times 320 \times 256$ & $12 D \times 16 D \times 12 D$ & $0.025 D\left(\approx 0.104 \delta_{99}\right)$ & $0.033 D$ \\
B(W) & $256 \times 320 \times 256$ & $16 D \times 16 D \times 16 D$ & $0.021 D\left(\approx 0.087 \delta_{99}\right)$ & $0.033 D$ \\
C & $384 \times 320 \times 384$ & $12 D \times 16 D \times 12 D$ & $0.017 D\left(\approx 0.070 \delta_{99}\right)$ & $0.033 D$ \\
C (W) & $384 \times 320 \times 384$ & $16 D \times 16 D \times 16 D$ & $0.014 D\left(\approx 0.058 \delta_{99}\right)$ & $0.033 D$ \\
\hline
\end{tabular}




\section{Non-Excited Jet}

The computations for the natural, non-excited jet provide a point of reference with respect to which the response of the excited jets can be interpreted. The computations were performed for Reynolds number $\mathrm{Re}=10^{4}$ defined based on the velocity of the jet, its diameter and the kinematic viscosity $(v)$ as $\operatorname{Re}=U_{j} D / v$. The inlet turbulence intensity was equal to $T i=0.01 U_{j}$ and the turbulent length scale needed for computing velocity fluctuations by using digital filtering method [32] was taken as $0.1 \mathrm{D}$.

A relatively low Reynolds number should enables accurate solutions on the mesh A which is utilised for the parametric study in Section 4.2. In the present case the accuracy of the results achieved on the mesh $\mathrm{A}$ is verified by comparison with results obtained using the finer grids and with literature data. Instantaneous results showing the natural jet visualised by an isosurface of the Q-parameter are presented in Fig. 4(a). The Q-parameter is defined as $Q=\frac{1}{2}\left(S_{i j} S_{i j}-\Omega_{i j} \Omega_{i j}\right)$ where $S_{i j}$ and $\Omega_{i j}$ are symmetrical and antisymmetrical parts of the velocity gradient tensor. In Fig. 4a the Q-parameter exhibits near the nozzle exit typical toroidal structures (rings) resulting from the Kelvin-Helmholtz instability followed by a vortex roll-up process. These structures break up at the end of the potential core, i.e., starting about $4 D-5 D$ from the inlet. The time-averaged contours of the axial velocity are presented in Fig. 4(b) on the right hand side. They exhibit a typical gradual widening of the jet as the flow develops downstream. Figure 5 shows instantaneous values of the normalised sub-grid viscosity $\left(v_{T} /\left(U_{j} D\right)\right)$ and also the time averaged sub-grid kinetic energy estimated using $k_{S G S}=\frac{v_{T}^{2}}{\left(C_{V} \Delta\right)^{2}}$ where $C_{V}=0.1$ [33] and $\Delta=(\Delta x \Delta y \Delta z)^{1 / 3}$ is the LES filter width. In all the cases presented in this paper the ratio of $k_{S G S}$ to the total turbulent kinetic energy is less than 0.1. Hence, according to Pope's criterion [34] the meshes used in the

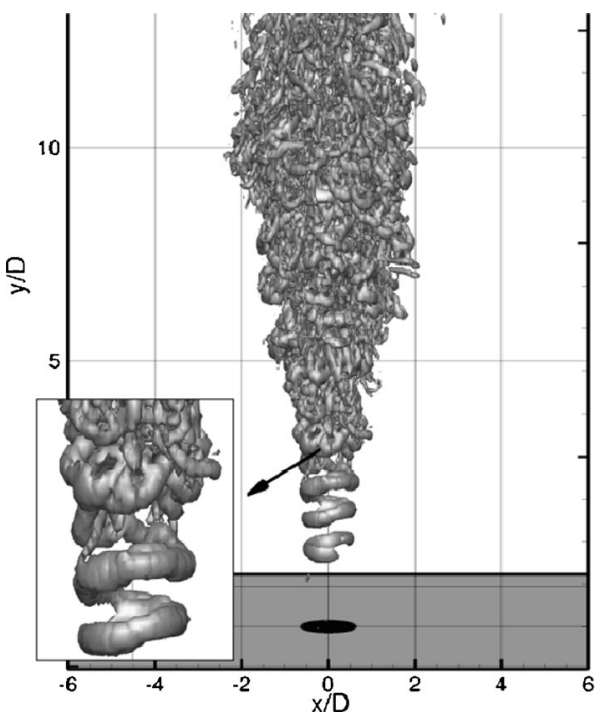

(a)

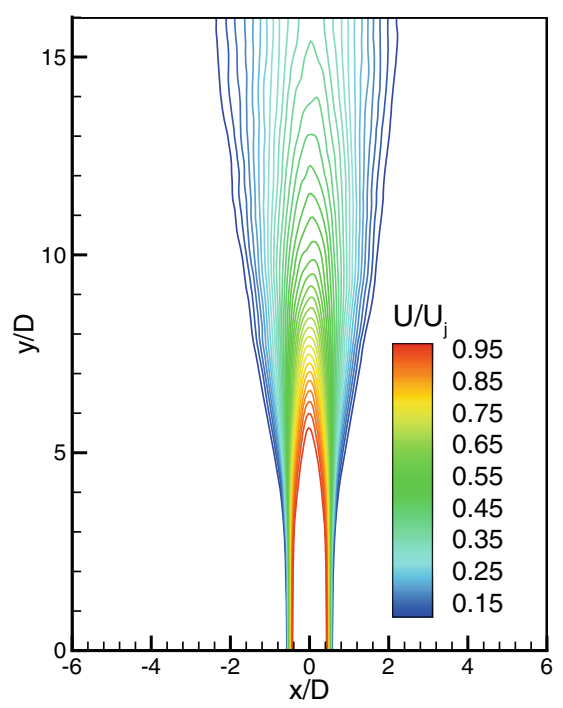

(b)

Fig. 4 Isosurface of the Q-parameter $\left(Q=0.5\left(U_{j} / D\right)^{2}\right)$ for a natural non-excited jet at $\operatorname{Re}=10^{4}$ (a) and contours of time-averaged normalised $\left(U / U_{j}\right)$ axial velocity (b) obtained on a mesh with $128 \times 160 \times 128$ nodes 
Fig. 5 Contours of the time averaged sub-grid kinetic energy (left part of the figure) and instantaneous value of the sub-grid viscosity. The results are for the natural non-excited jet at $\mathrm{Re}=10^{4}$ obtained on mesh A

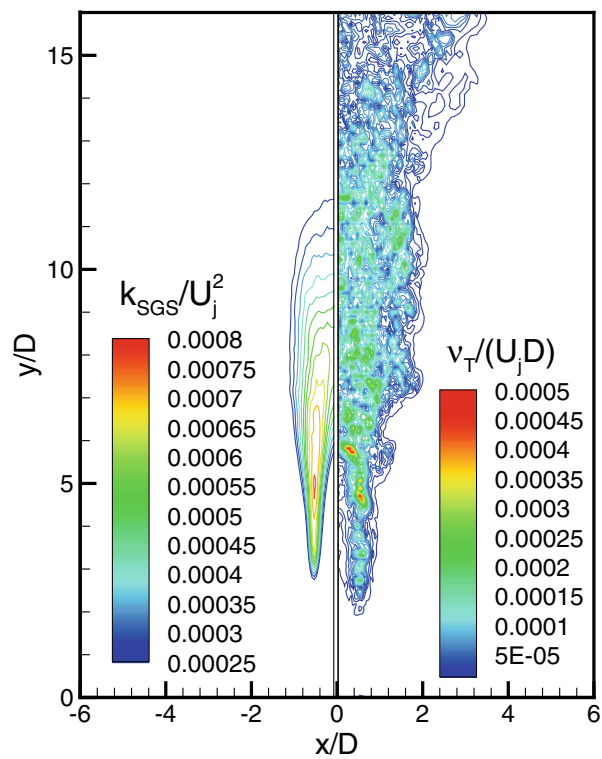

computations provide proper resolution. It is worth noting that the sub-grid model acts only when the flow becomes turbulent while in the initial region of the jet in the shear layer its influence is almost negligible - this is well seen in Fig. 5 noticing that the values $v_{T} /\left(U_{j} D\right)$ at $0<y / D<2$ is below $5 \times 10^{-5}$.

Figure 6 shows comparison of the present results (streamwise velocity) with experimental and numerical results taken from Refs. [2, 35]. Differences between the numerical results obtained on the reference and refined mesh are very small. Most likely this is due to the applied high-order discretization schemes which can give strong reductions of simulation errors upon grid refinement. It is seen that both the mean velocity and its fluctuations fit well within the range of literature data. In Fig. 6 it is seen that downstream of the potential core the mean velocity profiles differ somewhat from each other which is manifested by a faster or slower velocity decay. Discrepancies between the profiles of velocity fluctuations are seen closer to the inlet, i.e., from about $y / D=1$. These discrepancies concern the present results and all literature data and may be attributed to small differences in jet parameters at the inlet, i.e., mean velocity, turbulence characteristics and the Reynolds number. The influence of inlet conditions on the downstream jet evolution and self-similarity scaling for the far-field velocity is well known and was studied by many researchers. In [36] it was suggested that the jet behaviour in the far-field depends notably on the inlet conditions, consistent with the observed characteristic differences in Fig. 6.

\section{Bifurcations in Excited Jets}

A striking effect of axial and flapping forcing at the inflow of a turbulent jet is that under suitable conditions a qualitative change in the time-averaged structure of the flow emerges. Both bifurcations leading to a two-fold splitting and even a three-fold splitting will be discussed in this section, together with requirements on the forcing parameters that induce these transitions. In this section we first establish the accuracy of the computational model 


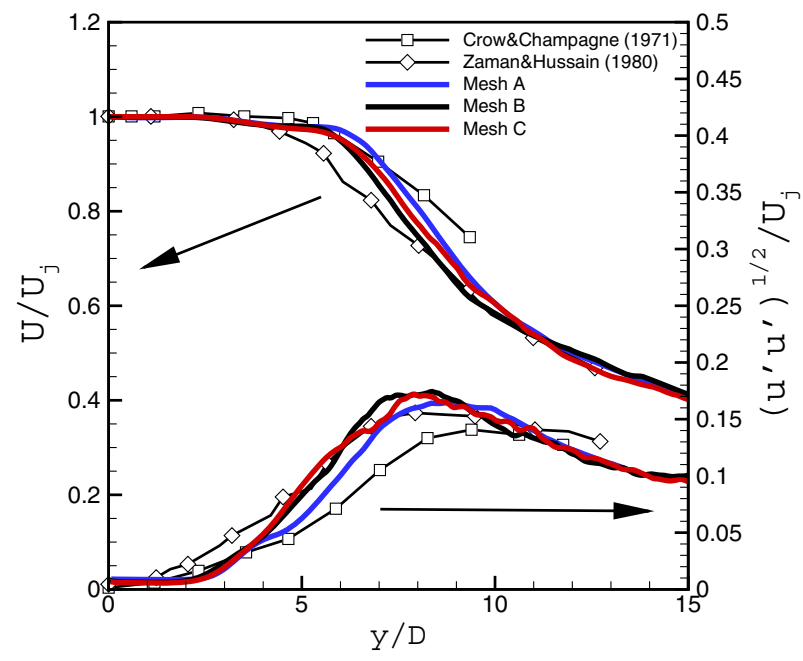

Fig. 6 Mean and fluctuating axial velocity profiles for natural turbulent jet. Literature data (lines with symbols) were obtained for $\operatorname{Re}=1.03 \times 10^{5}$ (Crow and Champagne [2]) and $\operatorname{Re}=3.2 \times 10^{4}$ (Zaman and Hussain [35]). The present results are for $\mathrm{Re}=10^{4}$

for flow at a modest Reynolds number $\mathrm{Re}=4300$ (Subsection 4.1) for which experimental results are available [8]. Subsequently, in Subsection 4.2 we consider an excited jet at $\operatorname{Re}=10^{4}$ for which the obtained results may be regarded as representative also for higher Reynolds numbers as the excited jets are only slightly dependent on Re [7]. The LES computations performed in [15] for $\operatorname{Re}=5 \times 10^{3}, 2.5 \times 10^{4}$ and $5 \times 10^{4}$ showed that the bifurcating jets are almost insensitive to Re.

The excitation parameters, as summarised in Table 2 . We analyse the influence of the excitation frequency and the phase shift between the axial and flapping excitation. We do this for a range of excitation amplitudes and input turbulence levels. The forcing frequencies and their ratio will be expressed by the Strouhal numbers $S t_{a}$ and $S t_{h}$ used in the definition of the forcing terms (3). All the cases reported in Table 2 were computed for $S t_{a}=0.35,0.40,0.45,0.50,0.55,0.60,0.65$. In this table the subscripts at $A_{1 \%}$ and $\mathrm{Ti}_{1 \%}$ denote the amplitude of the forcing or the level of natural turbulence intensity. The subscript at $\Phi_{0}$ stands for the phase shift (cf. Eq. (3)).

Table 2 Simulation parameters for excited jets with axial-flapping forcing. In the column for $S t_{a} / S t_{h}$ the indication 1, 2, 4 implies that computations were performed for $S t_{a} / S t_{h}=1, S t_{a} / S t_{h}=2$ and $S t_{a} / S t_{h}=4$

\begin{tabular}{lllll}
\hline Simulation & $A_{a}=A_{h}$ & $S t_{a} / S t_{h}$ & $\phi$ & $T i$ \\
\hline $\mathrm{A}_{1} \%-\Phi_{\frac{\pi}{4}}-\mathrm{Ti}_{1 \%}$ & $0.01 U_{j}$ & $1,2,4$ & $\pi / 4$ & $0.01 U_{j}$ \\
$\mathrm{~A}_{1} \%-\Phi_{0}-\mathrm{Ti}_{1} \%$ & $0.01 U_{j}$ & $1,2,4$ & 0 & $0.01 U_{j}$ \\
$\mathrm{~A}_{1} \%-\Phi_{0}-\mathrm{Ti}_{5} \%$ & $0.01 U_{j}$ & 2 & 0 & $0.05 U_{j}$ \\
$\mathrm{~A}_{5 \%}-\Phi_{\frac{\pi}{4}}-\mathrm{Ti}_{1 \%}$ & $0.05 U_{j}$ & $1,2,4$ & $\pi / 4$ & $0.01 U_{j}$ \\
$\mathrm{~A}_{5} \%-\Phi_{0}-\mathrm{Ti}_{1} \%$ & $0.05 U_{j}$ & $1,2,4$ & 0 & $0.01 U_{j}$ \\
$\mathrm{~A}_{5 \%}-\Phi_{0}-\mathrm{Ti}_{5} \%$ & $0.05 U_{j}$ & 2 & 0 & $0.05 U_{j}$ \\
\hline
\end{tabular}




\subsection{Forcing at $\operatorname{Re}=4300$}

To validate the computational approach we closely follow the set-up adopted in the experimental research presented in [8]. This concerns a large-amplitude excitation at $\mathrm{Re}=4300$ for which we take $A_{a}=A_{h}=0.15 U_{j}$. The excitation frequencies are taken as $S t_{a}=0.5$ and $S t_{a} / S t_{h}=2$. In the experiment [8] these excitation parameters led to a two-fold splitting of the jet and here we focus on whether or not this qualitative feature can be recovered accurately.

A very strong jet splitting is confirmed at the selected flow conditions, starting at $5 \mathrm{D}$ from the inlet, as is illustrated in Fig. 7. One may observe that the vortex rings visualised in Fig. 7a by the Q-parameter, are slightly inclined with respect to the inlet plane. This is the result of the flapping forcing which at the inlet pushes the vortex rings alternately from both sides. The successive vortices mutually interact, i.e., the vortex which is generated at the inlet amplifies the inclination of the proceeding vortex gradually increasing the inclination as the rings move away from the inlet.

At a distance of about $4 D$ the radial velocity becomes comparable to the axial velocity and the flow divides into two separate streams. This behaviour is further illustrated in Fig. 7b showing contours of the time-averaged axial velocity combined with the isosurface at value 0 of its instantaneous value. The axial velocity inside the rings is negative and its largest value reaches -0.2 , comparable with the forcing amplitude.

Comparison of time-averaged axial velocity profiles along the radial direction at four distances from the inlet $(y / D=4.0,5.0,6.5,8.0)$ obtained at the reference resolution (mesh A) and on two twice refined meshes (B and B(W)) are shown in Figs. 8 and 9 concerning results taken in the bifurcating plane and in Fig. 10 for the bisecting plane. Referring to Fig. 2 the bifurcating plane is the $x-y$ plane at $z=0$ whereas the bisecting plane is the $z-y$ plane at $x=0$. The figures 8 and 10 also include experimental results taken

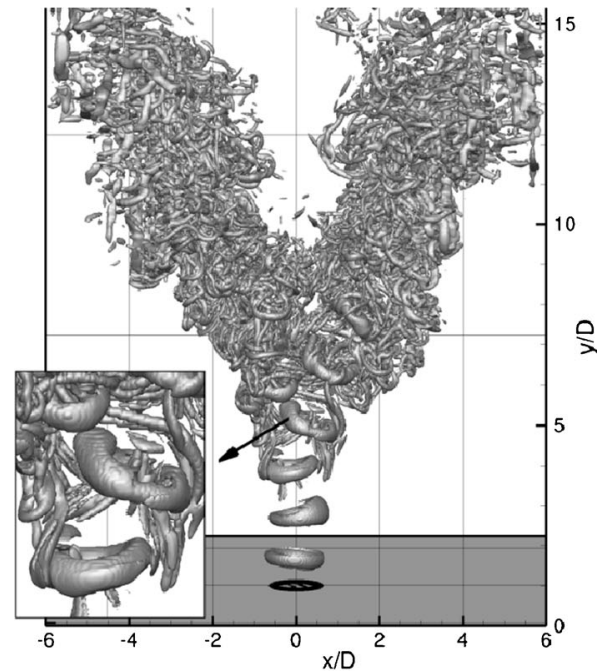

(a)

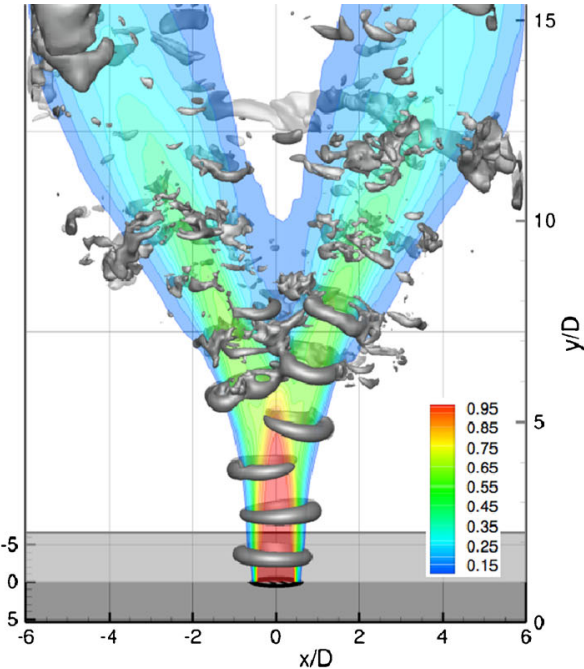

(b)

Fig. 7 Isosurface of the Q-parameter $\left(Q=0.5\left(U_{j} / D\right)^{2}\right)$ for an excited jet (a) and isosurface of the axial velocity $(u=0)$ together with contours of normalised time-averaged axial velocity $\left(U / U_{j}\right)(\mathrm{b})$. Axialflapping excitation was considered with $A_{a}=A_{h}=0.15 U_{j}, S t_{a}=0.5, S t_{a} / S t_{h}=2, \operatorname{Re}=4300$ 

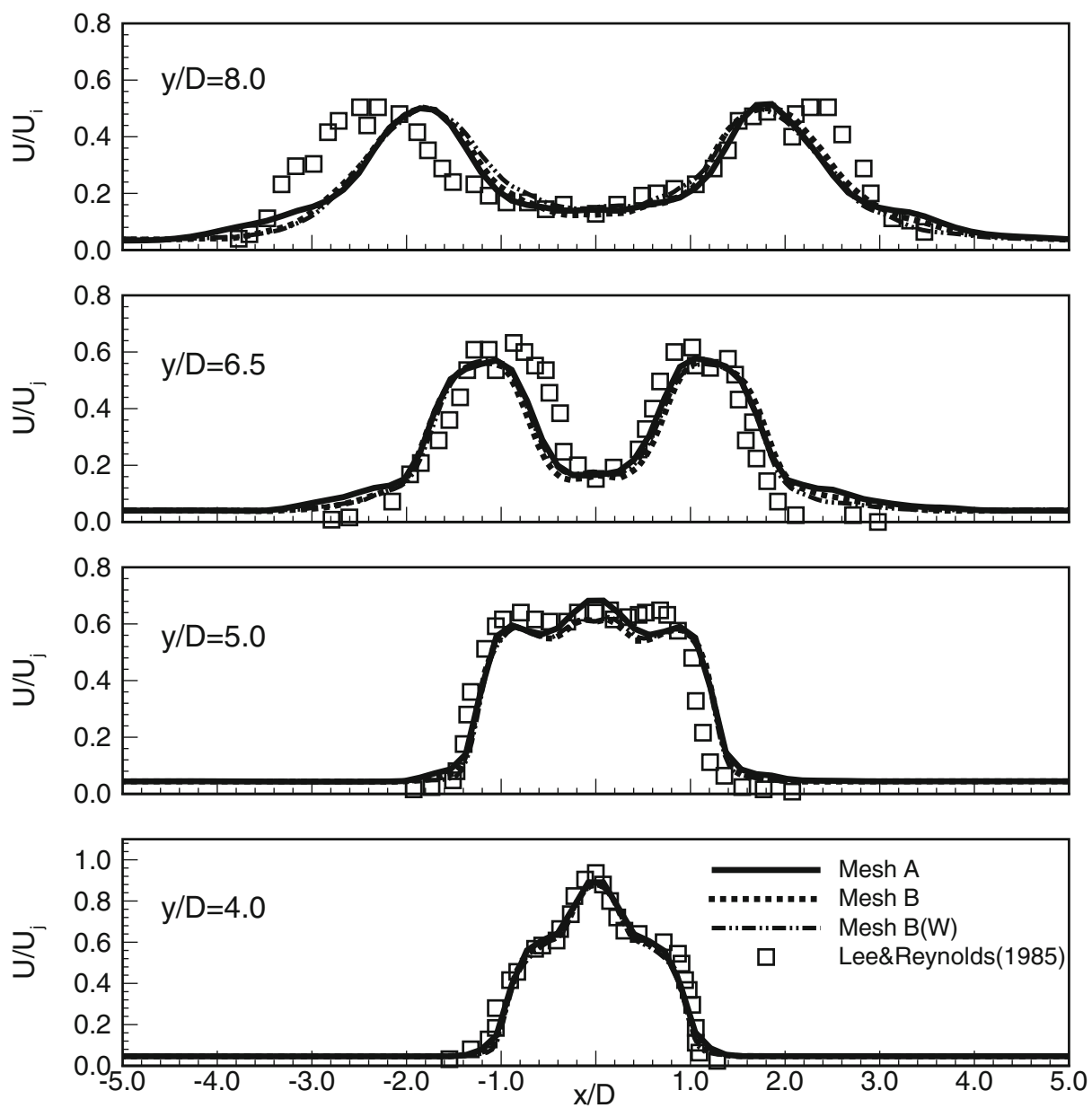

Fig. 8 Comparison of the axial velocity in the bifurcating plane for $\mathrm{Re}=4300$. Lines: present results obtained on the meshes A, B, B(W); symbols: experimental data from [8]

from [8]. The numerical results close to the inlet $(y / D=4.0,5.0)$ agree very well with the measurements; the values and shapes of the profiles of the axial velocity fit almost exactly the experimental results on all three tested grids. Downstream of the location where the jet divides in two streams the radial distance between the velocity maxima increases. Though the agreement with experimental data at $y / D=6.5,8.0$ is not as good as for $y / D=4.0,5.0$ the bifurcation phenomenon seems to be captured correctly. It should be noted that at $y / D=6.5,8.0$ the experimental data no longer strictly display symmetry with respect to the axis $x=0$. This indicates the accuracy with which the reference experimental data were obtained, placing the comparison in context.

Although a precise quantitative comparison is no longer possible far into the flow domain, the current setting of numerical parameters and flow conditions underpin the correct inclusion of the dominant flow physics in the simulations at the reference resolution of $128 \times 160 \times 128$ (mesh A). Moreover, the results obtained using the wider domain (mesh $\mathrm{B}(\mathrm{W})$ ) are only slightly different from those obtained using the basic computational domain. 

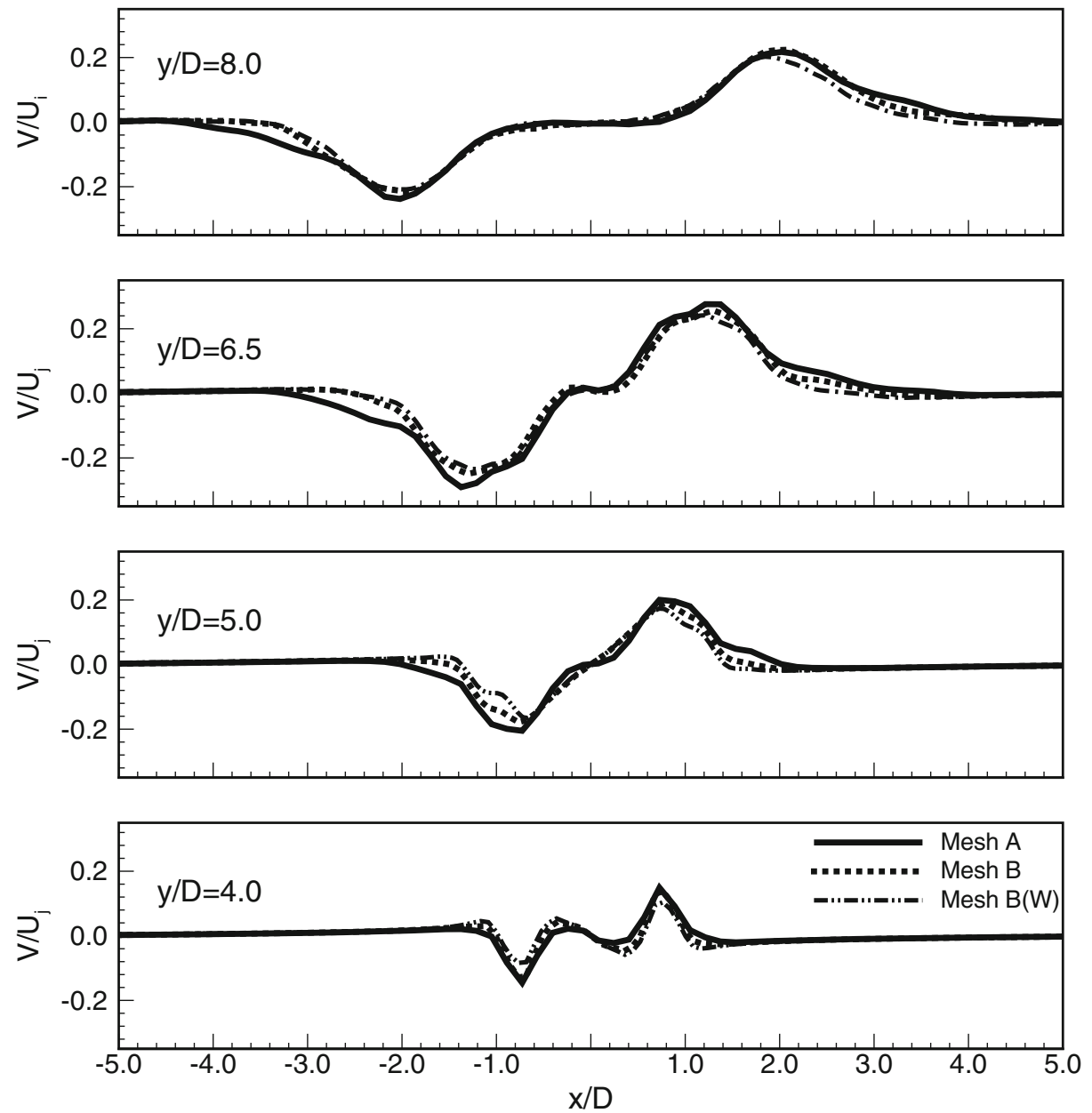

Fig. 9 Comparison of the horizontal velocity in the bifurcating plane for $\mathrm{Re}=4300$. Experimental data are not available

In Fig. 9 it is seen that at $y / D=8.0$ the horizontal velocity approaches zero at $x \approx 5 D$ and hence, one may assume that the influence of the side boundaries at $x, z= \pm 6 D$ on the region of the jet splitting is very small.

The results in the bisecting plane, presented in Fig. 10, show that the inlet velocity profiles exhibit a regular bell-shape. The radial spreading of the jet increases only very little whereas the maximum of the velocity decreases quickly; at $y / D=6.5$ it is equal to $0.2 U_{j}$ and at $y / D=8.0$ it is equal to $0.1 U_{j}$. The accuracy of the numerical solutions is confirmed by the agreement with the experimental data which show exactly the same behaviour on all applied grids.

\subsection{Forcing at $\operatorname{Re}=10^{4}$}

In the previous subsection we have shown that with the forcing parameters chosen according to literature the two-fold jet splitting phenomenon can be captured and the results of 

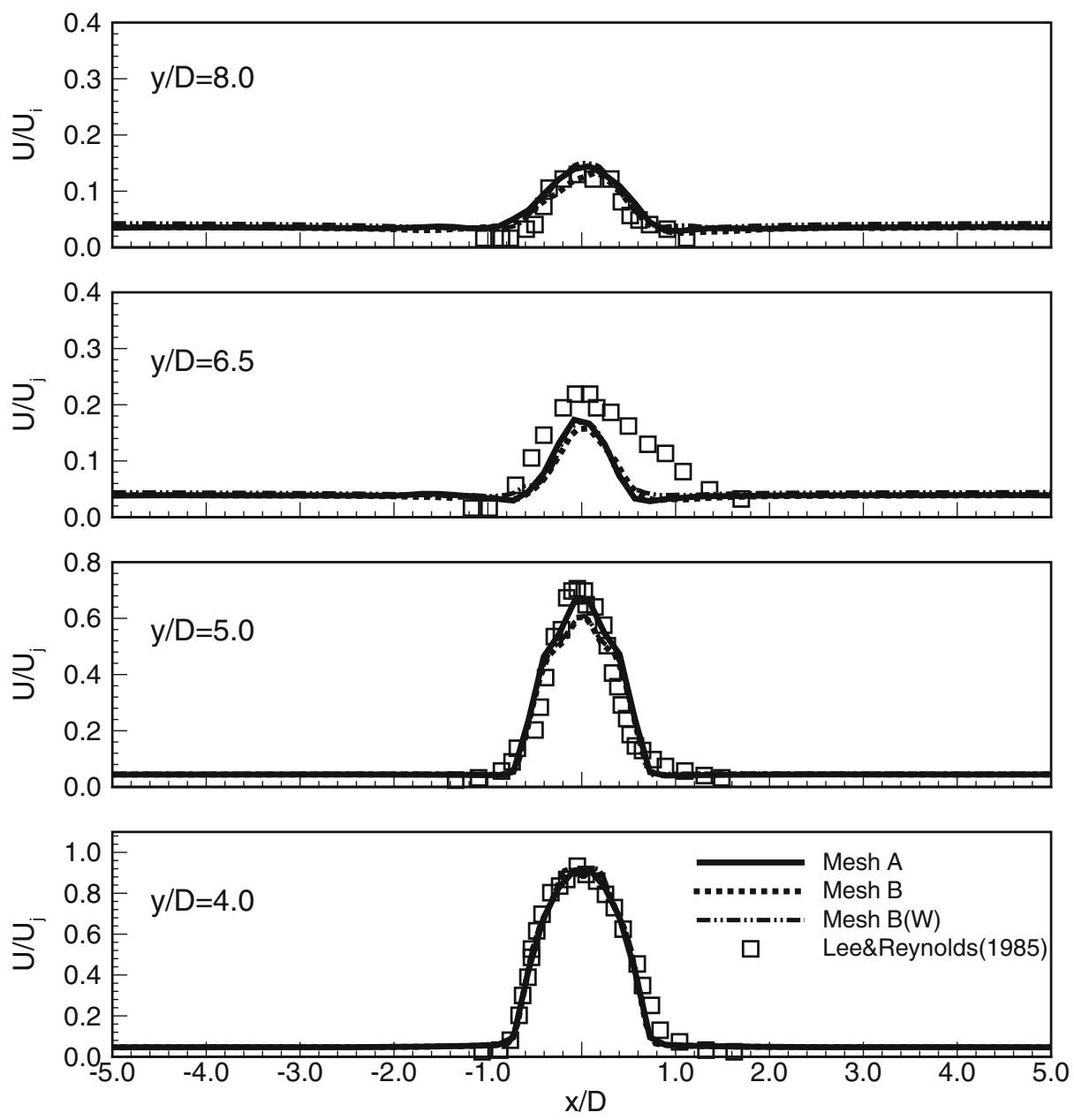

Fig. 10 Comparison of the axial velocity in the bisecting plane for $\operatorname{Re}=4300$. Lines: present results obtained on the meshes A, B, B(W); symbols: experimental data from [8]

simulations agree well with available experimental data using simulations on the reference. We now turn to forcing at a higher Reynolds number $\operatorname{Re}=10^{4}$. Some simulations were repeated at these flow conditions comparing the reference grid solutions achieved using mesh A with the results obtained on the refined meshes B, C and $\mathrm{C}(\mathrm{W})$. The test computations were performed for the setting corresponding to the case $\mathrm{A}_{5} \%-\Phi_{0}-\mathrm{Ti}_{1} \%$ with $S t_{a}=0.50$ and $S t_{a} / S t_{h}=2$. As will be shown in Sections 4.2 .3 for the chosen excitation parameters the jet clearly splits into two branches. Hence, the performed comparison may be regarded as characteristic also for other cases with a less complicated flow pattern. The profiles of the mean and fluctuating component of axial and horizontal velocity obtained from these computations are shown in Figs. 11, 12 and 13. These results correspond to the locations $y / D=3.0$ (upstream the splitting occuring at $y / D \approx 4.0$ ) and $y / D=7.0$ where two distinct jet branches exist. Firstly, it should be noted that the results obtained using the 

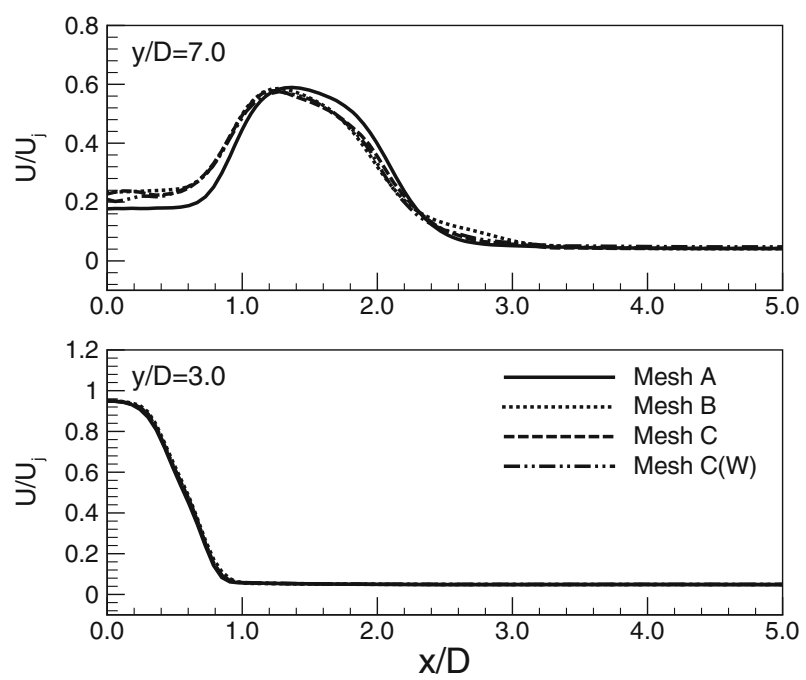

Fig. 11 Comparison of the mean axial velocity in the bifurcating plane for $\mathrm{Re}=10^{4}$ obtained on the meshes A, B, C and C(W). Case $\mathrm{A}_{5 \%}-\Phi_{0}-\mathrm{Ti}_{1} \%$ with $S t_{a}=0.50, S t_{a} / S t_{h}=2$

wider domain (mesh $\mathrm{C}(\mathrm{W})$ ) are in a good agreement with the solutions obtained using the basic computational domain. Such a behaviour is observed up to $y / D=11$ (not shown here) and therefore one may assume that up to this distance from the inlet the results are not noticeably influenced by the side boundaries. Secondly, it is seen that the results obtained on mesh A are close to those from the refined meshes. Some discrepancies are seen mainly
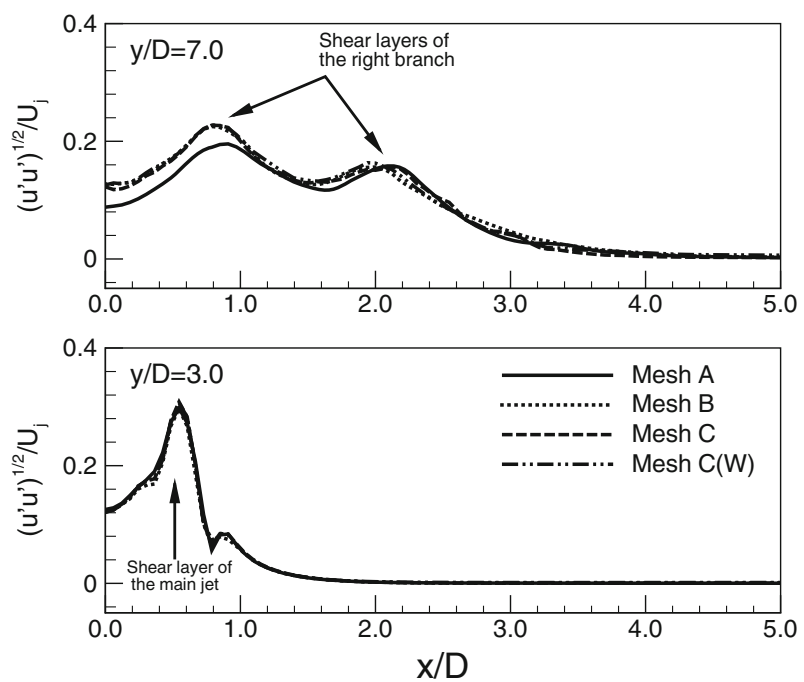

Fig. 12 Comparison of the fluctuating component of axial velocity in the bifurcating plane for $\operatorname{Re}=10^{4}$ obtained on the meshes A, B, C and C(W). Case $\mathrm{A}_{5} \%-\Phi_{0}-\mathrm{Ti}_{1} \%$ with $S t_{a}=0.50, S t_{a} / S t_{h}=2$. Arrows indicate local peaks related to the shear layers along the main jet at $y / D=3.0$ and at the right branch of the splitted jet at $y / D=7.0$ 

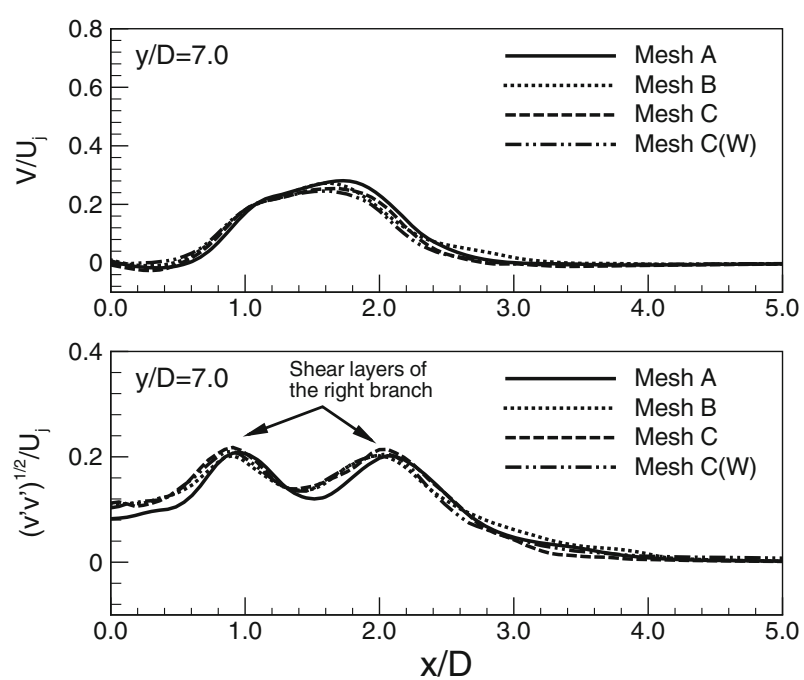

Fig. 13 Comparison of the mean and fluctuating components of horizontal velocity in the bifurcating plane for $\mathrm{Re}=10^{4}$ obtained on the meshes A, B, C and C(W). Case $\mathrm{A}_{5} \%-\Phi_{0}-\mathrm{Ti}_{1} \%$ with $S t_{a}=0.50, S t_{a} / S t_{h}=2$. Arrows indicate local peaks related to the shear layers along the right branch of the splitted jet

for $x / D \leq 1.0$ where the results from mesh A show a small underprediction. The fluctuating components (both $\left(u^{\prime} u^{\prime}\right)^{1 / 2} / U_{j}$ in Fig. 11 and $\left(v^{\prime} v^{\prime}\right)^{1 / 2} / U_{j}$ in Fig. 13) show existence of two local maxima occurring at the shear layers created along the branches of the jet. It is seen that mesh A enables accurate prediction of the levels and particularly the locations of these maxima. These findings confirm that also at the higher $R e$ value the coarse grid A yields reliable simulation findings, albeit with a slightly less well resolved range of smaller turbulent scales. Nevertheless, the main features of the flow can be well captured as far as the dominant response to the imposed perturbuations is concerned, e.g., jet splitting and spreading rates. The cost of the computations performed on the particular meshes differ substantially. For instance simulations up to time $400 D / U_{j}$ require 64 computing hours on mesh B using 32 Intel Xenon $2.9 \mathrm{GHz}$ processors and only 5.6 hours on mesh A using 16 processors, with quite comparable parallel performance. In the sequel of this paper we will therefore restrict ourselves to the grid A, thereby allowing to perform a comprehensive parameter study into effects of changes in several important system parameters such as forcing amplitudes, phase shifts and turbulence intensity at the inflow - according to the specification given in Table 2. The studies of the qualitative flow structuring at low and intermediate forcing levels and at different turbulence intensities are presented in separate subsections.

\subsubsection{Low amplitude forcing}

Low amplitude forcing is identified with $A_{a}=A_{h}=0.01 U_{j}$. In Table 2 these test cases are denoted as: $\mathrm{A}_{1 \%}-\Phi_{\frac{\pi}{4}}-\mathrm{Ti}_{1 \%}, \mathrm{~A}_{1 \%}-\Phi_{0}-\mathrm{Ti}_{1 \%}$ and $\mathrm{A}_{1 \%}-\Phi_{0}-\mathrm{Ti}_{5 \%}$. For $\mathrm{A}_{1 \%}-\Phi_{\frac{\pi}{4}}-\mathrm{Ti}_{1 \%}$ and $\mathrm{A}_{1 \%}-\Phi_{0}-\mathrm{Ti}_{1} \%$ the amplitude of the excitation is the same as the level of turbulence intensity whereas in the case $\mathrm{A}_{1 \%}-\Phi_{0}-\mathrm{Ti}_{5} \%$ the turbulence intensity is higher and equal to $T i=$ $0.05 U_{j}$. 
In case $S t_{a} / S t_{h}=1$, fig. 14 shows the contours of the time-averaged axial velocity in the bifurcating plane for $\mathrm{A}_{1 \%}-\Phi_{\frac{\pi}{4}}-\mathrm{Ti}_{1} \%$ at a variety of excitation frequencies. Evidently, no jetsplitting is obtained as seen in the previous subsection. Instead, these results show that the jet is deformed and asymmetric with respect to the axis. The observed asymmetry decreases when $S t_{a}$ increases. In fact, for $S t_{a} / S t_{h}=1$ the excitation as given by (3) is not symmetric, leading to the asymmetry in the downstream development. When the excitation frequencies are the same the term $\sin (\pi x / D)$ causes the flapping forcing to be subtracted from the axial forcing in case $x<0$, whereas for $x>0$ both forcing terms sum up constructively. Hence, the part of the vortex ring in $x>0$ is stronger than if $x<0$ and this leads to the asymmetry seen downstream.

In case with $S t_{a} / S t_{h}=4$, shown in Fig. 15, the bifurcation phenomenon is also absent, even if the flapping forcing acts on both sides of the jet alternately. Because the period of the flapping forcing is four times longer than the period of the axial excitation the flapping forcing interacts with pairs of two successive vortex rings in a similar manner - either pushes them or stops them. However, because the amplitude of the forcing is quite small the jets do not split and their shape remains similar to the natural jet. Moreover, as seen in Fig. 15, the solutions seem to be virtually independent of $S t_{a}$. A minor lack of symmetry comes rather from too modest averaging time and not from the asymmetric jet behaviour.

Turning to computations with $S t_{a} / S t_{h}=2$ a clear bifurcation leading to a jet splitting occurs. In this case the flapping forcing acts with successive vortex rings in such a way that it pushes and stops them alternately. From the results presented in Fig. 16 it is seen that the jet splitting is limited to a range of frequencies corresponding to $S t_{a}=0.4-0.55$. This range of agitation frequencies agrees well with experimental findings [6, 7]. One may observe that the angle of jet splitting, i.e., the angle between the jet branches, changes with $S t_{a}$ and its maximum is attained for $S t_{a} \approx 0.5$.

The influence of the phase difference between the axial and flapping forcing was investigated by considering simulations $\mathrm{A}_{1 \%}-\Phi_{0}-\mathrm{Ti}_{1} \%$ for which the axial and flapping excitation are in-phase (i.e., $\phi=0$ in (3)). Similarly as for $\mathrm{A}_{1 \%}-\Phi_{\frac{\pi}{4}}-\mathrm{Ti}_{1} \%$ the bifurcation phenomenon was observed only when $S t_{a} / S t_{h}=2$. For $S t_{a} / S t_{h}=1$ the jets were asymmetric whereas for $S t_{a} / S t_{h}=4$ the excitation played a rather insignificant role, at least from the point of view of large-scale deformation of the jets. The time-averaged results for the cases with
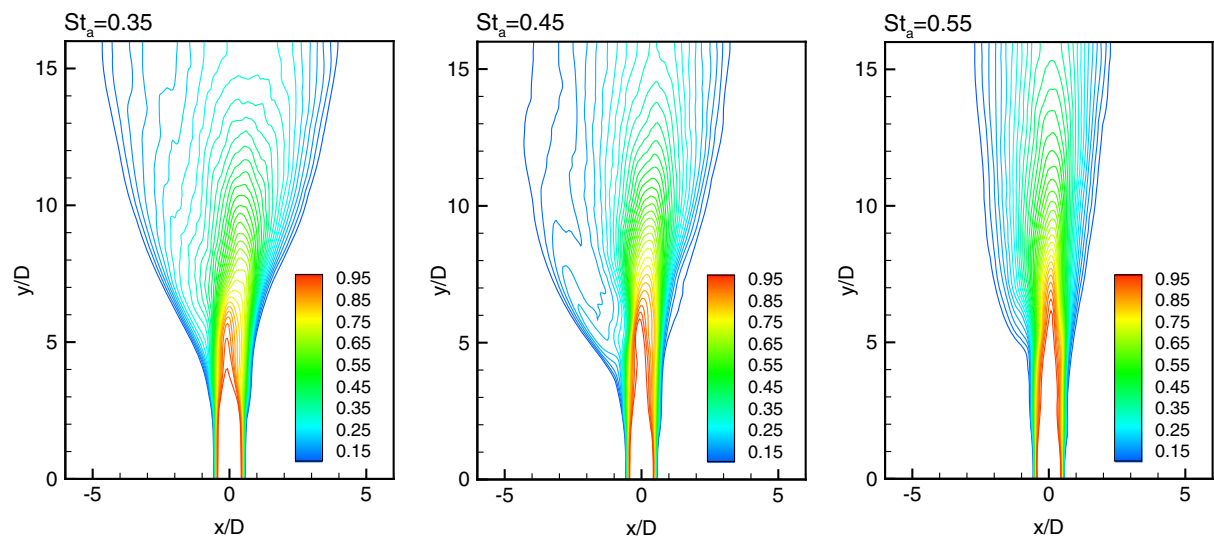

Fig. 14 Contours of the normalised time-averaged axial velocity $\left(U / U_{j}\right)$ in the bifurcation plane for simulations at $S t_{a}=0.35,0.45$ and 0.55 , using the setting indicated by: $\mathrm{A}_{1 \%}-\Phi_{\frac{\pi}{4}}-\mathrm{Ti}_{1} \%$ with $S t_{a} / S t_{h}=1$ 

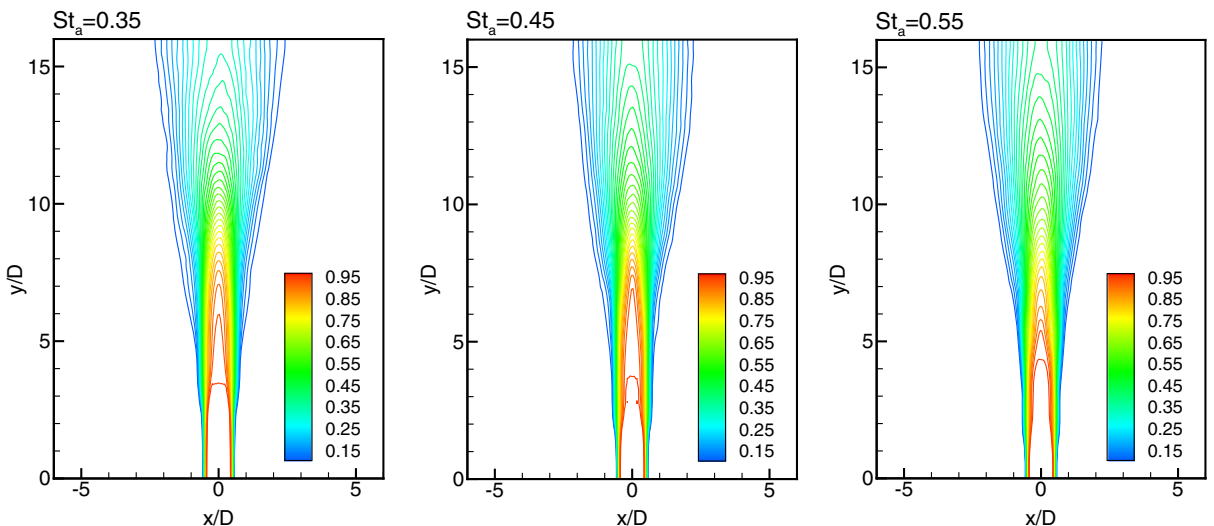

Fig. 15 Contours of the normalised time-averaged axial velocity $\left(U / U_{j}\right)$ in the bifurcation plane for simulations at $S t_{a}=0.35,0.45$ and 0.55 , using the setting indicated by: $\mathrm{A}_{1 \%}-\Phi_{\frac{\pi}{4}}-\mathrm{Ti}_{1 \%}$ with $S t_{a} / S t_{h}=4$
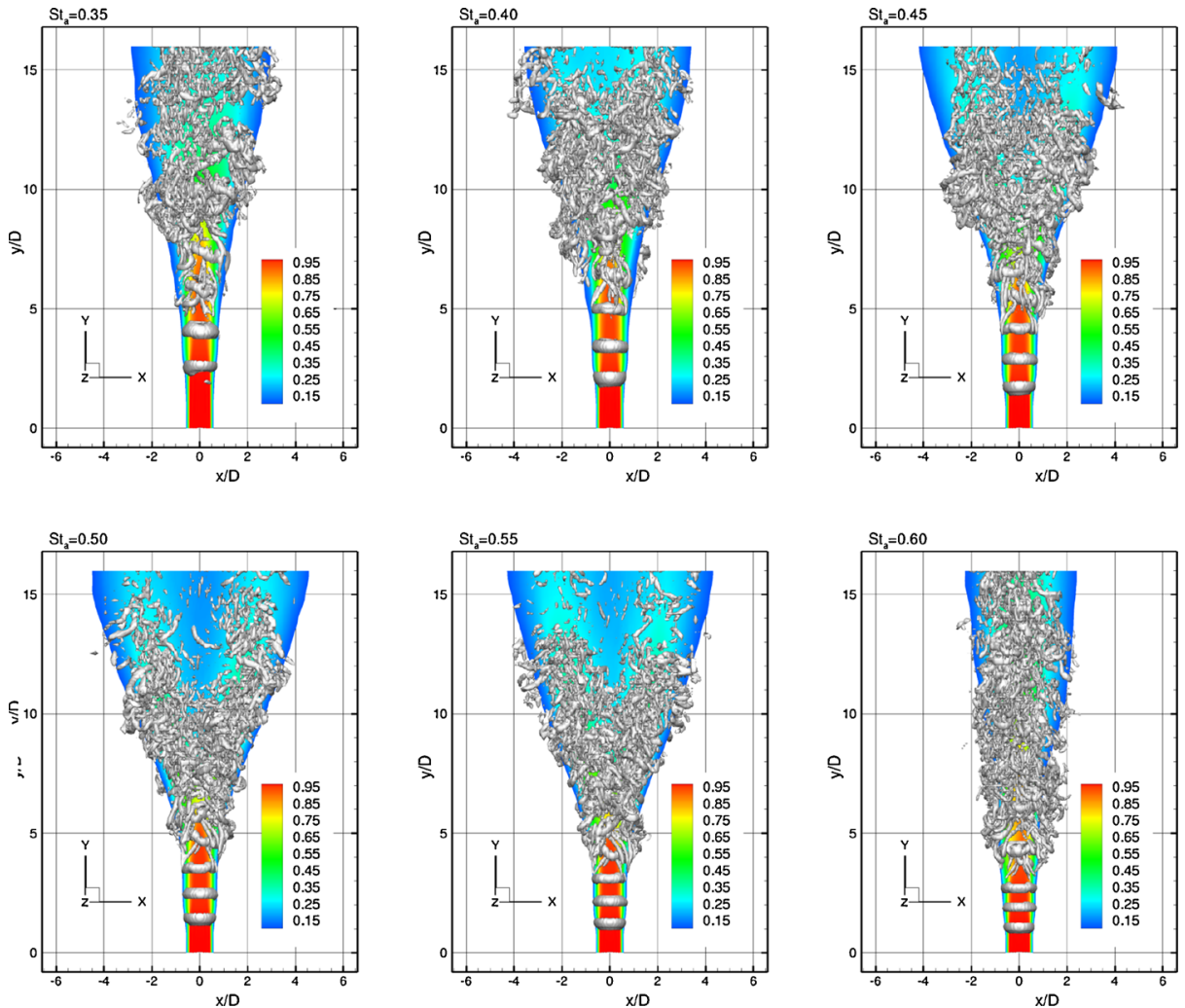

Fig. 16 Isosurface of instantaneous Q-parameter $\left(Q=0.5\left(U_{j} / D\right)^{2}\right)$ and contours of the normalised timeaveraged axial velocity $\left(U / U_{j}\right)$ in the bifurcation plane for simulations at $S t_{a}=0.35-0.60$, using the setting indicated by: $\mathrm{A}_{1 \%}-\Phi_{\frac{\pi}{4}}-\mathrm{Ti}_{1} \%$ with $S t_{a} / S t_{h}=2$. The jet splitting occurs in the cases with $S t_{a}=0.40-0.55$ 
$S t_{a} / S t_{h}=2$ are shown in Fig. 17. This figure should be compared with Fig. 16 showing the results obtained in simulations $\mathrm{A}_{1 \%}-\Phi_{\frac{\pi}{4}}-\mathrm{Ti}_{1} \%$. One may observe that the jet splitting for $\mathrm{A}_{1} \%-\Phi_{0}-\mathrm{Ti}_{1} \%$ is shifted to somewhat higher values of $S t_{a}$. If the excitation is such that $S t_{a}<0.45$ and for $S t_{a} \geq 0.65$ (not shown) the jets become distorted but do not bifurcate.

We also investigated the effect of increasing the turbulent intensity at the inflow. These involve simulations $\mathrm{A}_{1 \%}-\Phi_{0}-\mathrm{Ti}_{5 \%}$ for which the turbulence intensity was considerably higher than the excitation amplitudes $\left(T i=0.05 U_{j}\right.$ and $\left.A_{a}=A_{h}=0.01 U_{j}\right)$. In these simulations the effect of the axial and flapping excitations was very small and the jets seemed to be independent of the excitation frequency. Further tests appear to suggest that the forcing amplitudes must be higher than (or at least equal to) the level of $T i$ in order to possibly induce jet splitting. If this condition is not met the flow structures generated by the excitation do not grow while flowing downstream but rather are destroyed close to the inlet by the comparably strong turbulent fluctuations.

\subsubsection{Analysis in the spectral space}

Figure 18 shows the amplitude spectra of the axial velocity fluctuations corresponding to the simulations $\mathrm{A}_{1 \%}-\Phi_{\frac{\pi}{4}}-\mathrm{Ti}_{1} \%$ for the non-excited case as well as for $S t_{a} / S t_{h}=2$ with
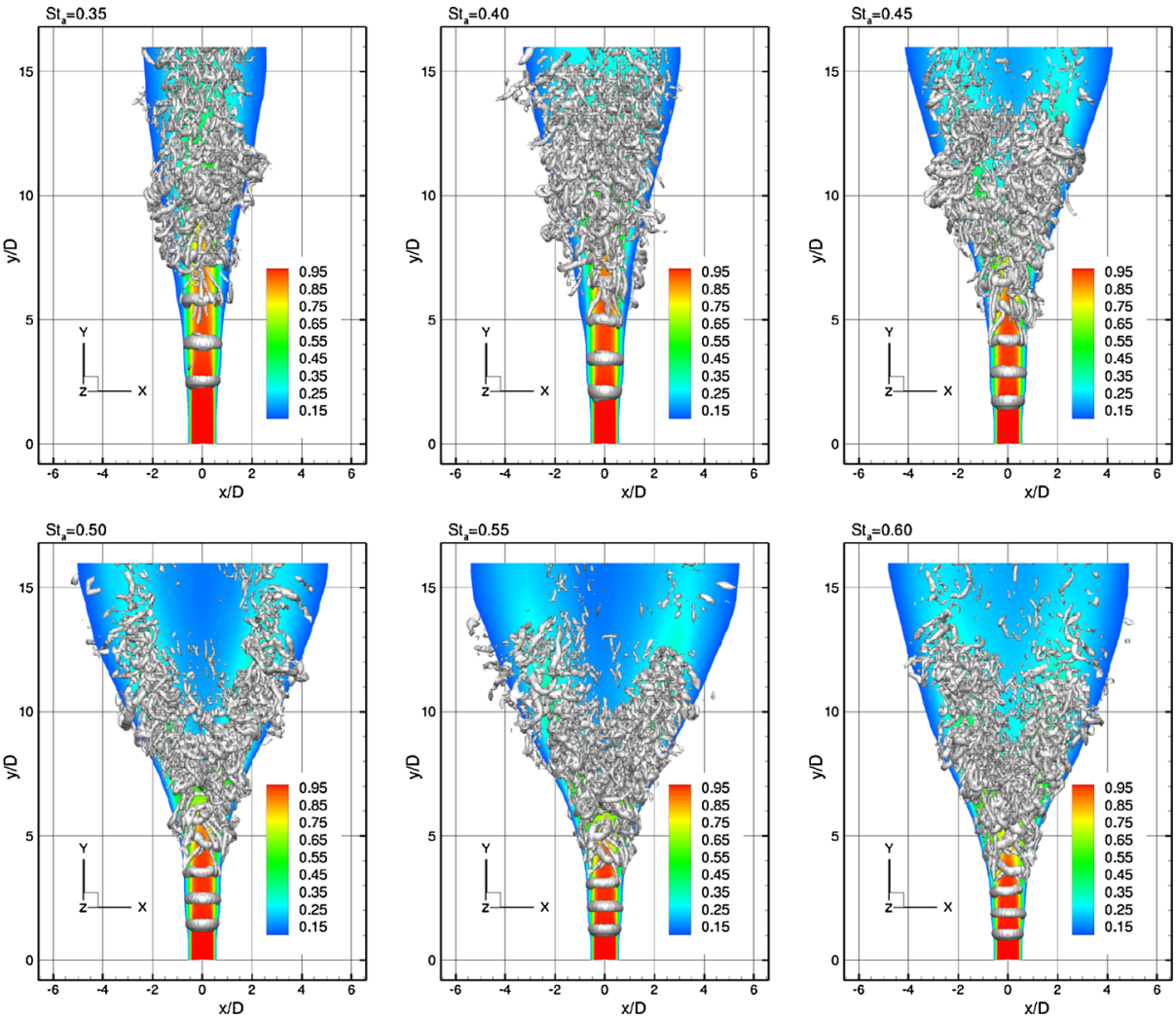

Fig. 17 Isosurface of instantaneous Q-parameter $\left(Q=0.5\left(U_{j} / D\right)^{2}\right)$ and contours of the normalised timeaveraged axial velocity $\left(U / U_{j}\right)$ in the bifurcation plane for simulations at $S t_{a}=0.35-0.60$, using the setting indicated by: $\mathrm{A}_{1 \%}-\Phi_{0}-\mathrm{Ti}_{1} \%$ with $S t_{a} / S t_{h}=2$. The jet splitting occurs in the cases with $S t_{a}=0.45-0.60$ 


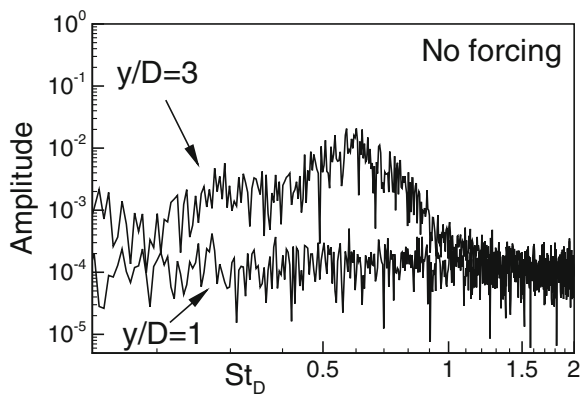

(a) no forcing

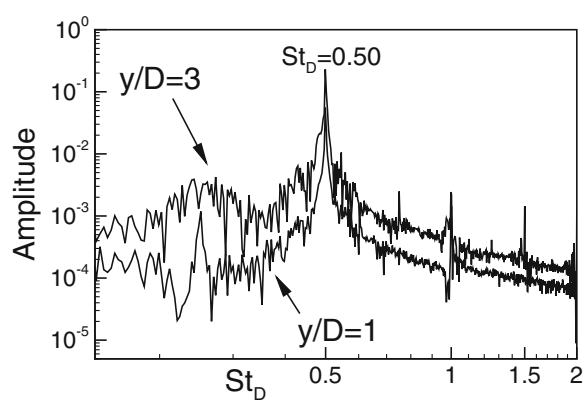

(c) $S t_{a}=0.50$

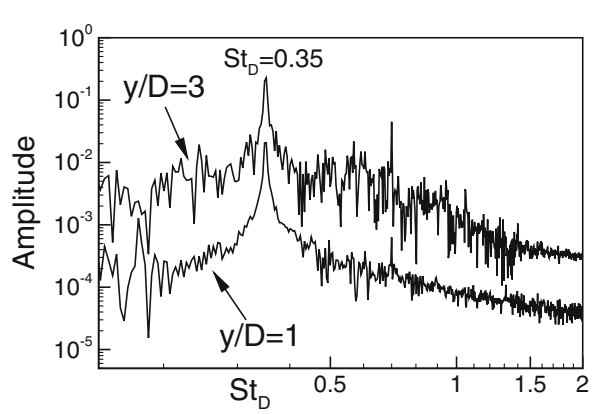

(b) $S t_{a}=0.35$

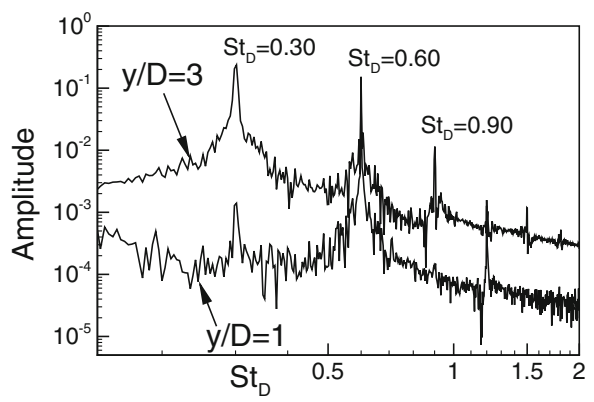

(d) $S t_{a}=0.60$

Fig. 18 Amplitude spectrum of the axial velocity fluctuations computed at the jet axis at $y / D=1$ and $y / D=3$. Results without excitation (a) and with excitations (b,c,d) with settings $\mathrm{A}_{1 \%}-\Phi_{\frac{\pi}{4}}-\mathrm{Ti}_{1 \%}$ with $S t_{a} / S t_{h}=2$ for $S t_{a}=0.35,0.5$ and 0.6 . The jet splitting occurs for $S t_{a}=0.5$

$S t_{a}=0.35,0.50,0.60$. These spectra were computed at the jet axis in two characteristic locations from the inlet, $y / D=1$ and $y / D=3$. The spectra are presented versus the Strouhal number $S t_{D}=f D / U_{j}$ where $f$ is the frequency of harmonics of the velocity signal. The non-excited jet shows no peaks in the spectrum which means that the "natural turbulence" case is free of any periodic forcing. At the distance $y / D=3$ a broadband region of intensified turbulence is observed, centered around $S t_{D}=0.58$ corresponding closely with the preferred mode frequency [37, 38].

In the cases involving excited jets at the inflow, we observe at $y / D=1$ distinct peaks corresponding directly to the axial excitation at the given $S t_{a}$. Additionally, at Strouhal numbers $S t_{D}=S t_{a} / 2$ one may also observe smaller peaks related to the flapping forcing. At a distance $y / D=3$ in the computations at $S t_{a}=0.5$ a very localized and pronounced peak is at $S t_{D}=0.5$ while for the remaining range of $S t_{D}$ the amplitude is low. The results obtained with $S t_{a}=0.35$ and $S t_{a}=0.6$ are qualitatively different from those at $S t_{a}=0.5$. In these cases the dominant peaks are seen at comparably low $S t_{D}$. Although the spectra display a similar broadband character and the peaks have similar level, they originate from different sources. In case $S t_{a}=0.35$ the observed peak is directly connected to the axial excitation, whereas for $S t_{a}=0.6$ the peak at $S t_{D}=0.3$ is partially due to the flapping forcing and partially due to the vortex pairing process related to the vortices generated by the axial excitation. Since the jet bifurcation was not seen for $S t_{a}=0.35$ nor $S t_{a}=0.6$ one may conclude that appearance of flow structures with the dominant frequencies much below the preferred mode frequency seems to prevent jet splitting. The 


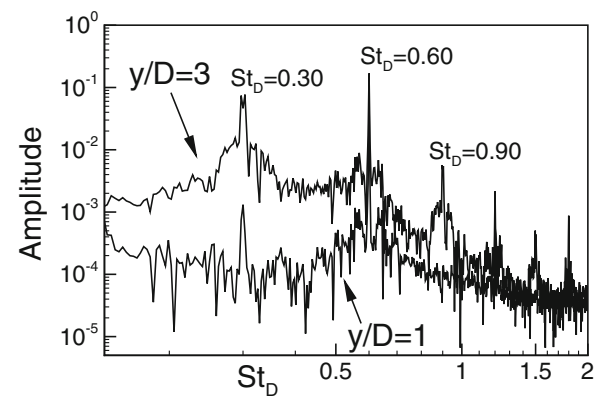

(a) $S t_{a}=0.60$

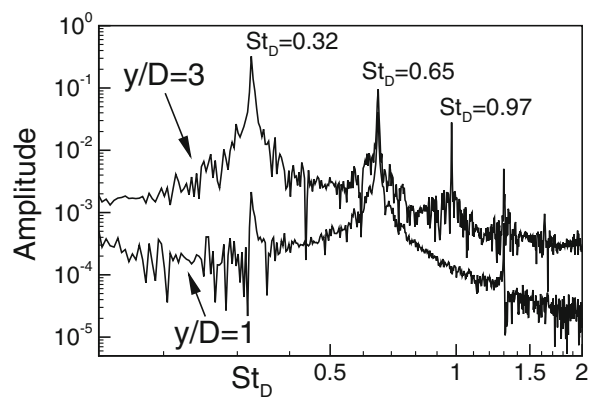

(b) $S t_{a}=0.65$

Fig. 19 Amplitude spectrum of the axial velocity fluctuations computed at the jet axis at $y / D=1$ and $y / D=3$. Test case $\mathrm{A}_{1 \%}-\Phi_{0}-\mathrm{Ti}_{1 \%}$ with $S t_{a} / S t_{h}=2$ for $S t_{a}=0.6$ and 0.65 . The jet splitting occurs for $S t_{a}=0.6$

jet appears to bifurcate only when close to the inflow region vortices initiate dominant frequency close to the preferred mode frequency. It is also confirmed by the results obtained for $\mathrm{A}_{1 \%}-\Phi_{0}-\mathrm{Ti}_{1 \%}$. The spectra for these simulations and $S t_{a}=0.60$ and $S t_{a}=0.65$ are shown in Fig. 19. It is seen that in both cases there are distinct peaks at higher harmonics and broadband regions around $S t_{D} \approx 0.3$. The subtle but very important difference between these spectra is that for $S t_{a}=0.65$ the observed low frequency peak is the highest one whereas for $S t_{a}=0.60$ the peak around $S t_{D} \approx 0.3$ is quite high but still smaller than the peak around $S t_{D} \approx 0.6$. In this case the jet was found to bifurcate whereas for $S t_{a}=0.65$ it does not.

\subsubsection{Medium amplitude forcing}

The results presented for the low amplitude forcing have shown that for weakly turbulent inlet conditions the combination of axial and flapping excitation may lead to jet bifurcation. This depends crucially on the choice of the excitation parameters, particularly $S t_{a}$ and the ratio $S t_{a} / S t_{h}$. Additionally, the magnitude of the amplitude of the excitations relative to the turbulence intensity is a key factor. It was shown that in order to create a bifurcating jet the excitation amplitudes need to be equal or larger than the turbulence intensity $T i$ at the inlet. In this section we consider cases with much bigger forcing amplitudes equal to $A_{a}=A_{h}=0.05 U_{j}$. We refer to these cases as 'medium' forcing amplitude and focus on two aspects: (i) differences between low and medium forcing amplitude; (ii) the range of parameters for which the bifurcation phenomenon occurs. The excitation amplitude $0.05 U_{j}$ is comparable to a turbulence intensity often occurring in jet flows studies, both in experimental and numerical works [39-43].

The results obtained for the medium forcing cases confirmed the findings from the previous subsections but also revealed some new flow features. Again, it turned out that to generate the two-fold jet splitting it is necessary that: $A_{a}=A_{h} \geq T i$. In the computations for the cases $\mathrm{A}_{5} \%-\Phi_{0}-\mathrm{Ti}_{1 \%}$ (i.e., with phase shift $\phi=0$ ) the forcing amplitudes are five times larger than $T i$ but even then jet splitting appeared only for $S t_{a} / S t_{h}=2$. For $S t_{a} / S t_{h}=1$ the jets became very asymmetric (particularly for low $S t_{a}$ ) while for $S t_{a} / S t_{h}=4$ only the spreading rate of the jets increased relative to the non-excited jet.

The results obtained in the simulations $\mathrm{A}_{5 \%}-\Phi_{\frac{\pi}{4}}-\mathrm{Ti}_{1 \%}$ (i.e., with $\phi=\frac{\pi}{4}$ ) with $S t_{a} / S t_{h}=$ 1 and $S t_{a} / S t_{h}=2$ are qualitatively similar to those obtained for $\mathrm{A}_{5} \%-\Phi_{0}-\mathrm{Ti}_{1 \%}$. For 
$S t_{a} / S t_{h}=1$ the jets were asymmetric and for $S t_{a} / S t_{h}=2$ the jets bifurcated - the results for $S t_{a}=0.40$ are shown in Fig. 20a,b. At $S t_{a} / S t_{h}=4$, we found that in a range of $S t_{a} \approx 0.45$ the jets also exhibited two-fold splitting in a similar way as in the cases with $S t_{a} / S t_{h}=2$. It was found that in these cases the bifurcation begun far from the inlet, at about $y / D=9$, and the angle between the jet branches is small as seen in Fig. 20c. Compared to the simulations with lower excitation amplitudes it turns out that with the larger excitations amplitudes the flapping excitation structures become strong enough to cause the jet separation further downstream, despite the factor four difference in time scale.

In the simulations $\mathrm{A}_{5} \%-\Phi_{0}-\mathrm{Ti}_{1 \%}$ and $\mathrm{A}_{5 \%}-\Phi_{\frac{\pi}{4}}-\mathrm{Ti}_{1 \%}$ with $S t_{a} / S t_{h}=2$ the bifurcation phenomenon was much stronger than at lower forcing amplitudes and the jet splitting was seen for almost the entire range of forcing frequencies investigated, i.e., $S t_{a}=0.35-$ 0.60 . A very interesting and surprising result was obtained in the simulation $\mathrm{A}_{5} \%-\Phi_{0}-\mathrm{Ti}_{1} \%$ with $S t_{a}=0.65$, where we noticed that the jet was split into three separate streams - the possible existence of this phenomenon was mentioned in [8] for triangular nozzles and also in [44] for an oscillating liquid jet issuing into the atmosphere. The triple jet splitting was revealed in the time-averaged results where the jet branching could be easily identified. The mean axial velocity contours obtained in these computations are shown in Fig. 21a. For comparison the results for the cases $\mathrm{A}_{5 \%}-\Phi_{\frac{\pi}{4}}-\mathrm{Ti}_{1} \%$ and $\mathrm{A}_{5 \%}-\Phi_{0}-\mathrm{Ti}_{5 \%}$ are presented in Fig. 21b,c. It is seen that for higher $T i$ or for $\phi=\pi / 4$ the jets do not divide, neither into two nor into three streams. From this example it appears that a jet with three branches is the effect of combination of three factors: careful selection of the forcing frequencies, sufficiently high forcing amplitudes with respect to $T i$ and consistency in phase of the axial and flapping forcing. Further research of three-fold branching of the jet is left for future studies.

Medium turbulence intensity - comparison of the axial velocity profiles In the computations $\mathrm{A}_{5 \%}-\Phi_{0}-\mathrm{Ti}_{5 \%}$ the bifurcation phenomenon was clearly seen for the range of frequencies $S t_{a}=0.45-0.60$. For $S t_{a} \leq 0.40$ the jets are affected only a little by the forcing, whereas for $S t_{a}=0.65$ only the spreading rate of the jet was increased. The axial velocity profiles obtained for $\mathrm{A}_{5 \%}-\Phi_{0}-\mathrm{Ti}_{5 \%}$ are shown in Fig. 22. The profiles are plotted along the centreline (Fig. 22a) and along the radial direction at the distance $y / D=10$ from

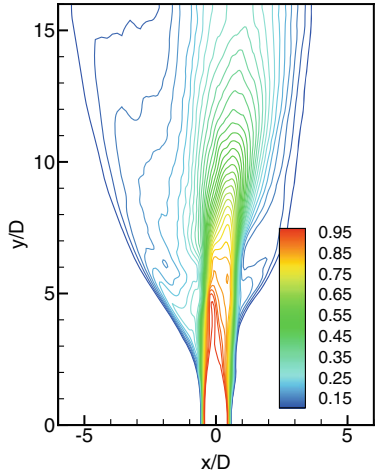

(a) $S t_{a} / S t_{h}=1$

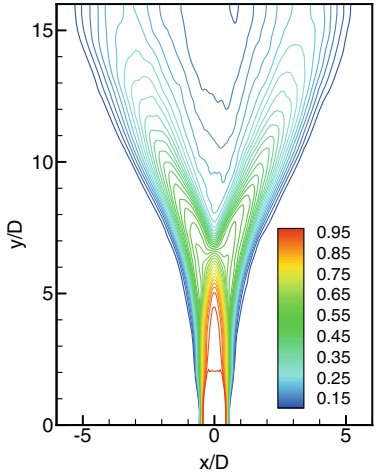

(b) $S t_{a} / S t_{h}=2$

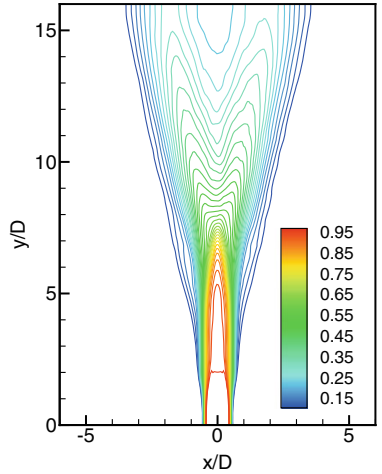

(c) $S t_{a} / S t_{h}=4$

Fig. 20 Contours of the time-averaged axial velocity in the bifurcation plane for simulations: $A_{5} \%-\Phi_{\frac{\pi}{4}}-T_{1} i_{1}$ for $S t_{a} / S t_{h}=1, S t_{a} / S t_{h}=2$ and $S t_{a} / S t_{h}=4$ with $S t_{a}=0.40$ 


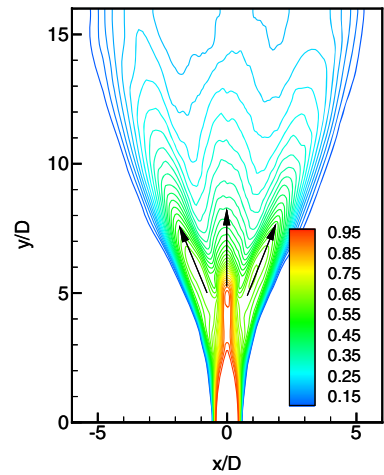

(a) $\mathrm{A}_{5 \%}-\Phi_{0}-\mathrm{Ti}_{1 \%}$

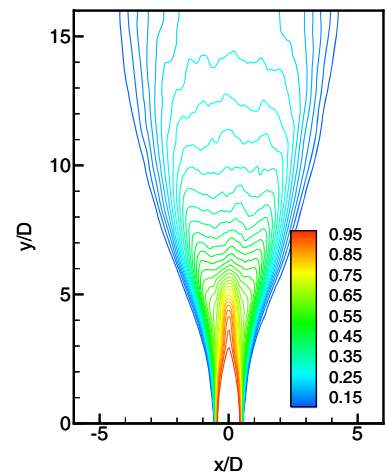

(b) $\mathrm{A}_{5 \%}-\Phi_{0}-\mathrm{Ti}_{5 \%}$

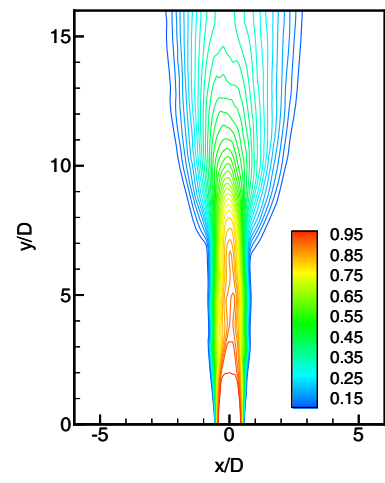

(c) $\mathrm{A}_{5 \%}-\Phi_{\frac{\pi}{4}}-\mathrm{Ti}_{1 \%}$

Fig. 21 Contours of the time-averaged axial velocity in the bifurcation plane for simulations: $\mathrm{A}_{5} \%-\Phi_{0}-\mathrm{Ti}_{1} \%$, $\mathrm{A}_{5} \%-\Phi_{0}-\mathrm{Ti}_{5} \%$ and $\mathrm{A}_{5 \%}-\Phi_{\frac{\pi}{4}}-\mathrm{Ti}_{1} \%$ with $S t_{a} / S t_{h}=2$ and $S t_{a}=0.65$. The arrows in figure (a) symbolically indicate three-fold branching

the inlet (Fig. 22b). From Fig. 22a it is seen that for all values of $S t_{a}$ the velocity along the jet axis smoothly decreases immediately after the inlet and there is no potential core where the natural unforced jets would maintain their initial velocity (Cf. Fig. 6). In the region up to $y / D=4$ the solutions are almost independent of $S t_{a}$, while evident differences appear only beyond $y / D=5$. In fact, the velocities decrease quickly with increasing $y / D$, particularly if $S t_{a}=0.50,0.55,0.60$. The profiles presented in Fig. 22a do not indicate whether jet bifurcation occurred or not. This is clear from the radial velocity profiles showing local maxima and minima of the streamwise velocity. In the following analysis the jet is regarded as bifurcated at some distance $y / D$ if the time-averaged axial velocity at the centreline is smaller than the time-averaged velocity at locations $x / D \neq 0$. As seen in Fig. 22b this condition is met in the simulations with $S t_{a}=0.35-0.60$, although for $S t_{a}=0.35,0.40$ the differences between the minima at the centreline $\left(U_{r, \min }\right)$ and maxima

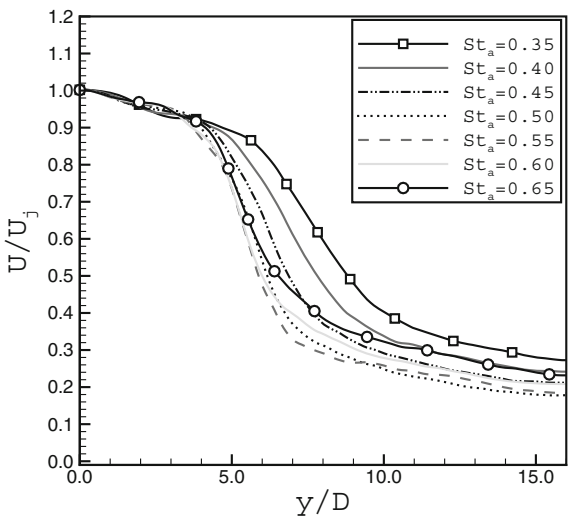

(a) centreline profiles

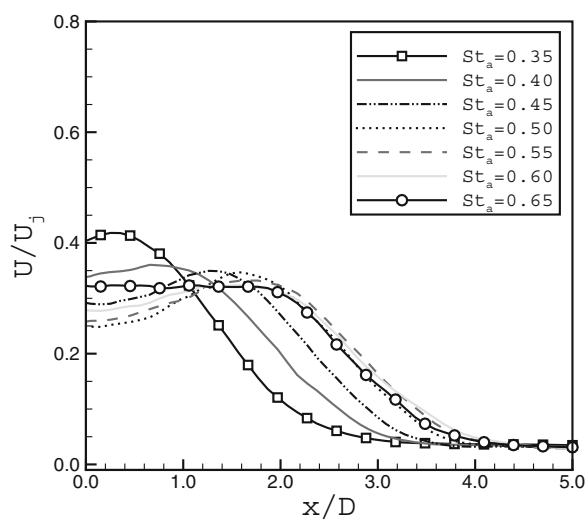

(b) radial profiles

Fig. 22 Mean axial velocity profiles along the centreline and in the radial direction in the bifurcation plane at $y / D=10$ for simulations $\mathrm{A}_{5 \%}-\Phi_{0}-\mathrm{Ti}_{5} \%$ with $S t_{a}=0.35-0.65$ and $S t_{a} / S t_{h}=2$ 
$\left(U_{r, \max }\right.$ at $x / D=0.35$ for $S t_{a}=0.35$ and $x / D=0.8$ for $\left.S t_{a}=0.40\right)$ are very small. In the following, the difference $\left(U_{r, \text { max }}-U_{r, \text { min }}\right)$ is used as a measure of the bifurcation strength. It is seen in Fig. 22b that in the simulations with $S t_{a}=0.35,0.40$ the values of $\left(U_{r, \max }-U_{r, \min }\right)$ are smaller than for $S t_{a}=0.45-0.60$. Moreover, the locations at which the maximal axial velocity is attained for $S t_{a}=0.45-0.60$ is clearly shifted from the centreline and occurs at $x / D=1.45-1.6$ approximately. Note that the forcing at $S t_{a}=0.45-0.60$ amplifies the frequencies lying in the range of the broadband preferred mode frequency (cf. Fig. 18) and this appears to lead to more pronounced jet splitting.

Low turbulence intensity - comparison of the axial velocity profiles. The simulations $\mathrm{A}_{5 \%}-\Phi_{0}-\mathrm{Ti}_{1} \%$ and $\mathrm{A}_{5 \%}-\Phi_{\frac{\pi}{4}}-\mathrm{Ti}_{1 \%}$, i.e., with relatively modest turbulence levels exhibit the existence of strong jet bifurcations. The profiles of the axial velocity at the centreline and along the radial direction are shown in Fig. 23. The profiles at the centreline up to $y / D<3$ are practically independent of $S t_{a}$ for both phase differences; in this region the axial velocity decreases smoothly. Around $y / D=3.5-4.0$ some of the profiles exhibit a very strong

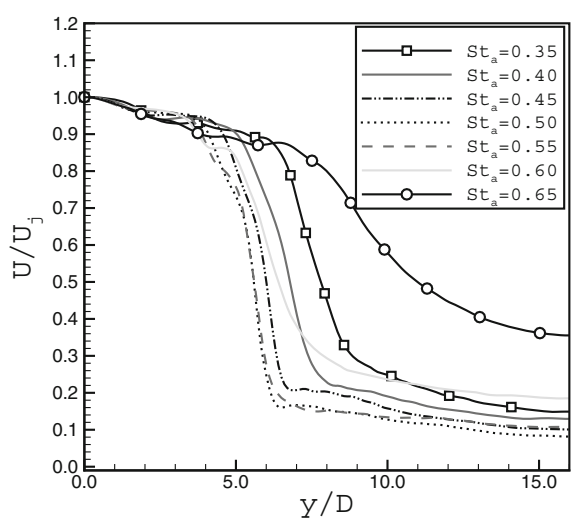

(a) centreline profiles for $\mathrm{A}_{5 \%}-\Phi_{\frac{\pi}{4}}-\mathrm{Ti}_{1} \%$

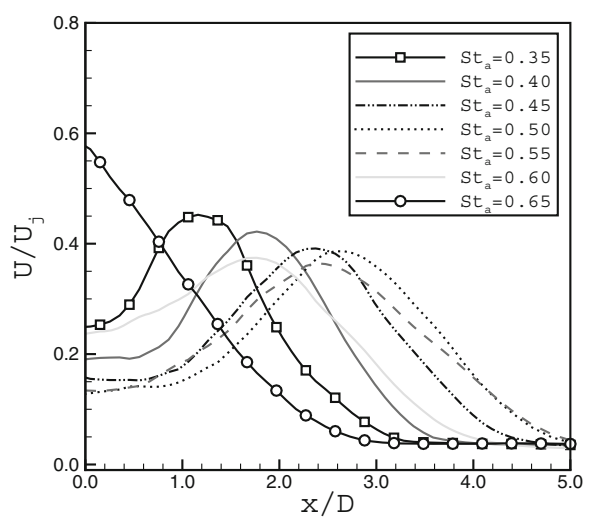

(c) radial profiles for $\mathrm{A}_{5 \%}-\Phi_{\frac{\pi}{4}}-\mathrm{Ti}_{1 \%}$

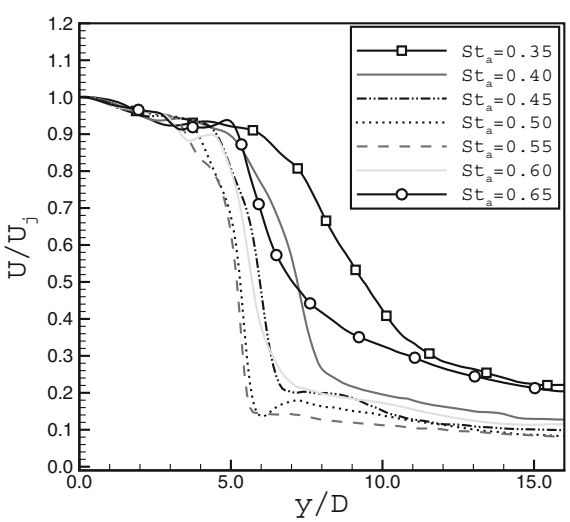

(b) centreline profiles for $A_{5 \%}-\Phi_{0}-T i_{1 \%}$

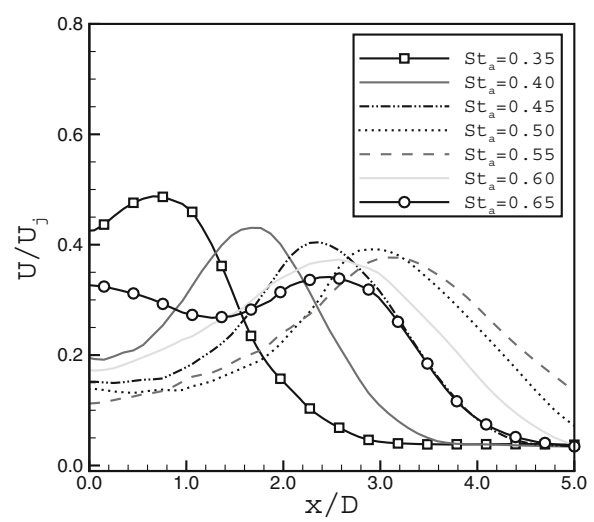

(d) radial profiles for $A_{5 \%}-\Phi_{0}-T_{1} \%$

Fig. 23 Mean axial velocity profiles along the centreline and in the radial direction in the bifurcation plane at $y / D=10$ for simulations: $\mathrm{A}_{5} \%-\Phi_{\frac{\pi}{4}}-\mathrm{Ti}_{1} \%$ and $\mathrm{A}_{5} \%-\Phi_{0}-\mathrm{Ti}_{1} \%$ with $S t_{a}=0.35-0.65$ and $S t_{a} / S t_{h}=2$ 
Fig. 24 Radial profiles of the time-averaged axial velocity taken at $y / D=7, \ldots, 13$ from the inlet. Simulation $\mathrm{A}_{5 \%}-\Phi_{\frac{\pi}{4}}-\mathrm{Ti}_{1 \%}, S t_{a}=0.50$, $S t_{a} / S t_{h}=2$

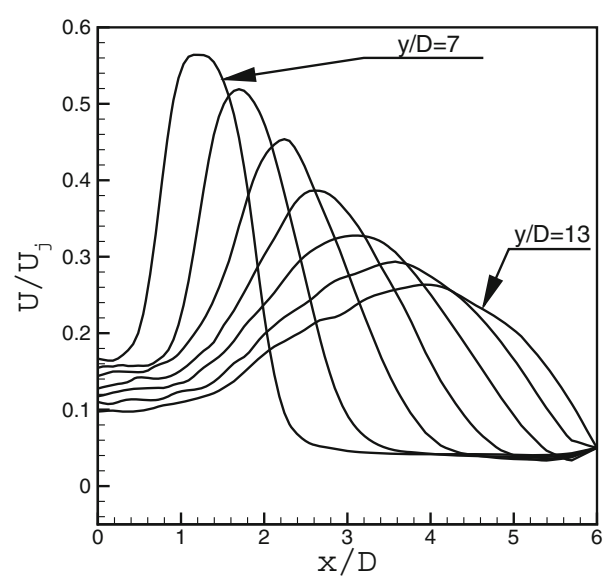

and sudden drop of the velocity, related directly to the jet bifurcation. The results obtained for $S t_{a}=0.50,0.55$ show the fastest decrease of the velocity - from a value 0.9 at $y / D=4$ to 0.1 at $y / D \approx 5.5$.

The radial profiles of the velocity presented in Fig. 23c,d show that for $S t_{a}=0.40-0.60$ the jets behave similarly and the differences between $\mathrm{A}_{5} \%-\Phi_{\frac{\pi}{4}}-\mathrm{Ti}_{1 \%}$ and $\mathrm{A}_{5} \%-\Phi_{0}-\mathrm{Ti}_{1} \%$ are only quantitative. The values of the streamwise velocity at the centreline are similar. The maxima in the streamwise direction show interesting differences in the location at which these are attained. For simulations $\mathrm{A}_{5 \%}-\Phi_{\frac{\pi}{4}}-\mathrm{Ti}_{1 \%}$ (see Fig. 23c) the location at which $U_{r, \max }$ is assumed reaches up to $x / D=2.8$ at an excitation with $S t_{a}=0.5$. Analogous results for $\mathrm{A}_{5 \%}-\Phi_{0}-\mathrm{Ti}_{1 \%}$ (see Fig. 23d) indicate the location of the maximum at $x / D=3.2$. Additionally, for $\mathrm{A}_{5 \%}-\Phi_{0}-\mathrm{Ti}_{1} \%$ the branches of the jet expand radially much more than for $\mathrm{A}_{5 \%}-\Phi_{\frac{\pi}{4}}-\mathrm{Ti}_{1 \%}$.

The most pronounced differences between $\mathrm{A}_{5} \%-\Phi_{\frac{\pi}{4}}-\mathrm{Ti}_{1} \%$ and $\mathrm{A}_{5} \%-\Phi_{0}-\mathrm{Ti}_{1 \%}$ are seen for $S t_{a}=0.65$ - in this case the phase shift changes the situation also qualitatively. Computations with excitation at $\phi=0$ exhibit three-fold splitting, which is absent in case when the

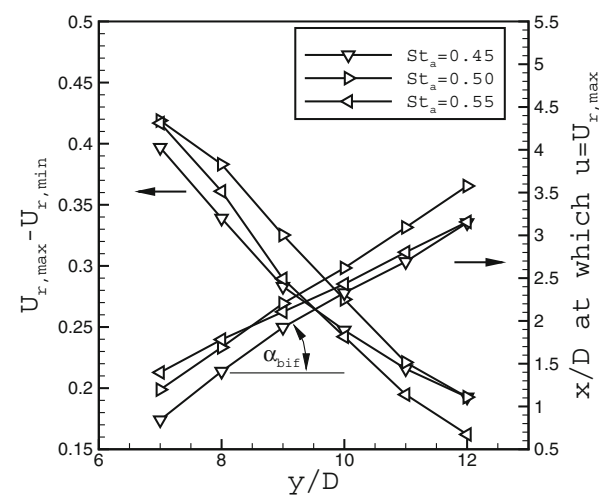

(a) $\mathrm{A}_{5 \%}-\Phi_{\frac{\pi}{4}}-\mathrm{Ti}_{1 \%}$

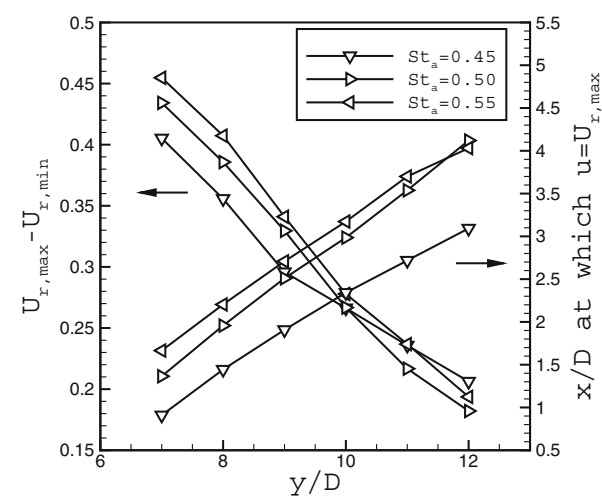

(b) $\mathrm{A}_{5 \%}-\Phi_{0}-\mathrm{Ti}_{1 \%}$

Fig. 25 Radial location of the maximum velocity $\left(U_{r, \max }\right)$ and values of $U_{r, \max }-U_{r, \min }$ for simulations: $\mathrm{A}_{5 \%}-\Phi_{\frac{\pi}{4}}-\mathrm{Ti}_{1} \%$ and $\mathrm{A}_{5 \%}-\Phi_{0}-\mathrm{Ti}_{1} \%$ with $S t_{a} / S t_{h}=2$ 
Table 3 Bifurcation angle obtained form simulations $\mathrm{A}_{5 \%}-\Phi_{\frac{\pi}{4}}-\mathrm{Ti}_{1 \%}$ and $\mathrm{A}_{5 \%}-\Phi_{0}-\mathrm{Ti}_{1 \%}$ with $S t_{a} / S t_{h}=2$

\begin{tabular}{lll}
\hline$S t_{a}$ & $\mathrm{~A}_{5 \%}-\Phi_{0}-\mathrm{Ti}_{1 \%}$ & $\mathrm{~A}_{5 \%}-\Phi_{\frac{\pi}{4}}-\mathrm{Ti}_{1 \%}$ \\
\hline 0.40 & 20.9 & 22.1 \\
0.45 & 23.0 & 22.8 \\
0.50 & 27.8 & 25.4 \\
0.55 & 26.5 & 18.7 \\
0.60 & 17.7 & 9.6 \\
\hline
\end{tabular}

phase shift is set to $\phi=\pi / 4$. Three-fold splitting was not reported in literature thus far for the selected excitation methods, analysis of this case is left for future studies.

\subsubsection{Bifurcation strength and bifurcation angle}

Finally, we analyse the evolution of the axial velocity profiles as function of $y / D$ distance. Figure 24 shows its radial profiles at $y / D=7,8, \ldots, 13$. These results were obtained for the case $\mathrm{A}_{5 \%}-\Phi_{\frac{\pi}{4}}-\mathrm{Ti}_{1} \%$ with $S t_{a}=0.5$ and $S t_{a} / S t_{h}=2$. It is seen that at $y / D=$ 7 the maximum and minimum velocity $\left(U_{r, \max }\right.$ and $\left.U_{r, \min }\right)$ take the highest values and $U_{r, \max }$ is located closest to the centerline axis. Further from the inlet the location of the maximum moves radially outward while the values of both $U_{r, \max }$ and $U_{r, \min }$ decrease. The differences $\left(U_{r, \text { max }}-U_{r, \text { min }}\right)$ for the simulations $\mathrm{A}_{5 \%}-\Phi_{\frac{\pi}{4}}-\mathrm{Ti}_{1} \%$ and for $\mathrm{A}_{5 \%}-\Phi_{0}-\mathrm{Ti}_{1 \%}$ are presented in Fig. 25. It is seen that for $\mathrm{A}_{5} \%-\Phi_{\frac{\pi}{4}}-\mathrm{Ti}_{1 \%}$ the values $\left(U_{r, \text { max }}-U_{r, \text { min }}\right)$ are smaller than for $\mathrm{A}_{5 \%}-\Phi_{0}-\mathrm{Ti}_{1 \%}$, implying that in the latter case the bifurcation is stronger, particularly for $S t_{a}=0.50,0.55$. The figure Fig. 25 shows also the lines corresponding to the locations at which $u=U_{r, \max }$. The nearly linear behaviour of these lines allowed to compute the angle $\left(\alpha_{b i f}\right)$ between the jet branches. This angle (called the bifurcation angle) was estimated based on a straight line between solutions at $y / D=8$ and $y / D=11$ for which the location at which $u=U_{r, \max }$ was far from the domain boundary and also from the centreline where the flow stagnates. The angle $\alpha_{b i f}$ is symbolically shown in Fig. 25a and its detailed values are reported in Table 3. The maximum values of $\alpha_{b i f}$ occur for $S t_{a}=0.50$, both for $\mathrm{A}_{5} \%-\Phi_{0}-\mathrm{Ti}_{1} \%$ and $\mathrm{A}_{5} \%-\Phi_{\frac{\pi}{4}}-\mathrm{Ti}_{1 \%}$. For the excitation at $S t_{a}<0.5$ the differences in $\alpha_{b i f}$ are small and seem to be only weakly dependent on $S t_{a}$. On the other hand for $S t_{a}>0.5$ the values of $\alpha_{b i f}$ obtained for $\mathrm{A}_{5 \%}-\Phi_{0}-\mathrm{Ti}_{1 \%}$ and $\mathrm{A}_{5} \%-\Phi_{\frac{\pi}{4}}-\mathrm{Ti}_{1 \%}$ differ substantially. For the simulation $\mathrm{A}_{5} \%-\Phi_{0}-\mathrm{Ti}_{1} \%$ at $S t_{a}=0.60$, the bifurcation angle is almost twice larger than for simulation $\mathrm{A}_{5} \%-\Phi_{\frac{\pi}{4}}-\mathrm{Ti}_{1} \%$. Hence, taking into account $\alpha_{b i f}$ one may say that for the higher forcing frequencies the excitation with $\phi=0$ is more efficient.

\section{Conclusions}

The paper presented a computational analysis of excited turbulent jets with low and medium amplitude axial and flapping excitations, operating with varying forcing frequencies. The results were validated against literature data for the natural, non-excited jet and also for the bifurcating jet at low Reynolds number 4300. The high-order numerical code allowed to obtain accurate results at the selected spatial resolution, which enabled computations for a large number of the cases thereby quantifying the dependence of the dynamic behavior on the parameters that characterize the external forcing. The numerical results confirmed the existing knowledge concerning the bifurcating jets and also revealed new findings, particularly quantifying forcing levels relative to turbulence intensities such that jet splitting could 
be observed at properly selected agitation frequencies, and even establishing a three-fold jet splitting under correct forcing conditions.

The energy input needed to generate a bifurcating jet was found to be higher or at least comparable to the inlet turbulence intensity. If this is not the case the natural turbulence destroys the modulated vortices and a well-known turbulent jet profile would emerge. In cases where the difference between the excitation amplitudes and the turbulence intensity increases the range of forcing frequencies for which jet splitting arises also increases. Additionally, it was observed that if the forcing amplitude is significantly higher than $T i$ (in one of the simulated cases five times) the jet splitting occurs not only for a forcing frequency ratio $S t_{a} / S t_{h}=2$ as reported in literature, but also for $S t_{a} / S t_{h}=4$. The strength of this additional jet splitting is lower than in case $S t_{a} / S t_{h}=2$, and also the range of forcing frequencies for which such splitting was observed was more limited.

The influence of a phase shift between the axial and flapping forcing on the jet dynamics was also investigated. In general it was found that if the axial and flapping forcing are perfectly in phase then jet splitting occurs at larger $S t_{a}$. Also, in these cases the angle of bifurcation and the strength of the bifurcation are larger. A very interesting jet behaviour was observed for cases with high forcing amplitudes and low turbulence intensities. In these simulations for forcing frequencies $S t_{a} \leq 0.6$ the jets exhibited the classical two-fold splitting but when $S t_{a}$ was increased further to 0.65 a three-fold branching appeared. This effect is subject to ongoing research and further analysis will be conducted and published elsewhere.

Acknowledgments This work was partially funded within the statutory funds BS/PB-1-103-3010/11/P and the research grant DEC-2011/03/B/ST8/06401. The authors acknowledge Prof. A. Boguslawski for fruitful discussions and help in the interpretation of the results. The authors are grateful to the computing centres SARA (The Netherlands, project SH-061) and Cyfronet PL-Grid Infrastructure (Poland) where the computations were carried out.

\section{References}

1. Kral L.D.: Active flow control technology, Technical Brief, ASME Fluids Engineering Division (1998)

2. Crow, S.C., Champagne, F.H.: Orderly Structure in Jet Turbulence. J. Fluid Mech. 48, 547-691 (1971)

3. Vreman, B., Geurts, B., Kuerten, H.: Discretization error dominance over subgrid terms in large eddy simulation of compressible shear layers in 2D. Commun. Numer. Methods Eng. 10(10), 785-790 (1994)

4. Geurts, B.J., Fröhlich, J.: Numerical effects contaminating les; a mixed story. In: Geurts, B.J. (ed.): Modern Simulation Strategies for Turbulent Flow, Vol. 317, pp. 309-330 (2001). Edwards, R. T.

5. Geurts, B.J., Fröhlich, J.: A framework for predicting accuracy limitations in large-eddy simulation. Phys. Fluids 14, L41 (2002)

6. Lee, M., Reynolds, W.C.: Bifurcating and blooming jets. technical report TF-22. Stanford University (1985)

7. Parekh, D., Leonard, A., Reynolds, W.C.: Bifurcating jets at high reynolds number. technical report TF-35. Stanford University (1988)

8. Reynolds, W.C., Parekh, D.E., Juvet, P.J.D., Lee, M.J.D.: Bifurcating and Blooming Jets. Ann. Rev. Fluid Mech. 35, 295-315 (2003)

9. Ball, C.G., Fellouah, H., Pollard, A.: The flow field in turbulent round free jets. Prog. Aerosp. Sci. 50, 1-26 (2012)

10. Freund, J.B., Moin, P.: Mixing enhancement in jet exhaust using fluidic actuators: direct numerical simulations. ASME: FEDSM98-5235 (1998)

11. Freund, J.B., Moin, P.: Jet mixing enhancement by high amplitude fluidic actuation. AIAA J. 38(10), 1863 (2000)

12. Danaila, I., Boersma, B.J.: Direct numerical simulation of bifurcating jets. Phys. Fluids 12(5), 12551257 (1999)

13. Hilgers, A., Boersma, B.J.: Optimization of turbulent jet mixing. Fluid Dyn. Res. 29, 345-368 (2001) 
14. Urbin, G., Metais, O.: Direct and large eddy simulations ii, chapter large-eddy simulations of threedimensional spatially developing round jets. Kluwer Academic Publishers (1997)

15. da Silva, C.B., Metais, O.: Vortex control of bifurcating jets: a numerical study. Phys. Fluids 14(11), 3798-3819 (2002)

16. Tyliszczak, A., Boguslawski, A.: LES of variable density bifurcating jets. In: Iacarrino, G., Kassinos, S.C., Langer, C.A., Moin, P. (eds.): Complex Effects in Large Eddy Simulations, in Lecture Notes in Computational Science and Engineering, Vol. 56, pp. 273-288. Springer (2007)

17. Heuveline, V.: On higher-order mixed fem for low mach number: application to a natural convection benchmark problem. Int. J. Numer. Methods Fluids 41, 1339-1356 (2003)

18. Knikker, R.: A comparative study of high-order variable-property segregated algorithms for unsteady low mach number flows. Int. J. Numer. Methods Fluids 66, 403-427 (2011)

19. Geurts, B.J.: Elements of Direct and Large-Eddy Simulation. R.T. Edwards, Philadelphia, PA (2003)

20. Sagaut, P.: Large eddy simulation for incompressible flows. Springer, New York (2001)

21. Vreman, A.W.: An Eddy-Viscosity subgrid-scale model for turbulent shear flow: algebraic theory and applications. Phys. Fluids 16, 3670-3681 (2004)

22. Kuban, L., Laval, J.P., Elsner, W., Tyliszczak, A., Marquillie, M.: LES modeling of converging-diverging turbulent channel flow. J. Turbul. 13, 1-19 (2012)

23. Tyliszczak, A., Boguslawski, A., Drobniak, S.: Quality of les predictions of isothermal and hot round jet. Qual.Reliab. Large Eddy Simul., ERCOFTAC Ser. 12, 259-270 (2008)

24. Boguslawski, A., Tyliszczak, A., Drobniak, S., Asendrych, D.: Self-sustained oscillations in a homogeneous-density round jet. J. Turbul. 14(4), 25-52 (2013)

25. Aniszewski, W., Boguslawski, A., Marek, M., Tyliszczak, A.: A new approach to sub-grid surface tension for les of two-phase flows. J. Comput. Phys. 231, 7368-7397 (2012)

26. Tyliszczak, A.: Assessment of implementation variants of conditional scalar dissipation rate in les-cmc simulation of auto-ignition of hydrogen jet. Arch. Mech. 65, 97-129 (2013)

27. Tyliszczak, A.: LES-CMC and les-flamelet simulation of non-premixed methane flame (Sandia F). J. Theor. Appl. Mech. 51, 859-871 (2013)

28. Pitsch, H., Steiner, H.: Large-eddy simulation of a turbulent piloted methane/air diffusion flame (Sandia Flame D). Phys. Fluids 12(10), 2541-2554 (2000)

29. Laizet, S., Lamballais, E.: High-order compact schemes for incompressible flows: a simple and efficient method with quasi-spectral accuracy. J. Comput. Phys. 228, 5989-6015 (2009)

30. Suzuki, Y., Suzuki, H., Kasagi, N.: Active control of an axisymetric jet with distributed electromagnetic flap actuators. Exp. Fluids 36, 498-509 (2004)

31. Sadeghi, H., Pollard, A.: Effects of passive control rings positioned in the shear layer and potential core of a turbulent round jet. Phys. Fluids 115103, 24 (2012)

32. Klein, M., Sadiki, A., Janicka, J.: A digital filter based generation of inflow data for spatially developing direct numerical and large eddy simulations. J. Comput. Phys. 186, 652-665 (2003)

33. Pope, S.B.: Turbulent flows. Cambridge University Press (2000)

34. Pope, S.B.: Ten questions concerning the large-eddy simulation of turbulent flows. New J. Phys. 6(35) (2004)

35. Zaman, K.B.M.Q., Hussain, A.K.M.F.: Vortex pairing in a circular jet under controlled excitation. J. Fluid Mech. 101, 449-491 (1980)

36. Nieuwstadt, F.T.M., Boersma, B.J., Bethouwer, G.: A numerical investigation on the effect of the inflow conditions on the self-similar region of a round jet. Phys. Fluids 10, 899-909 (1998)

37. Sadeghi, H., Pollard, A.: The preferred and shear layer modes in a jet under passive controls.proceedings of 18th australasian fluid mechanics conference launceston, Australia (2012)

38. Gutmark, E., Ho, C.M.: Preferred modes and the spreading rates of jets. Phys. Fluids 26, 2932-2938 (1983)

39. Antonia, R.A., Zhao, Q.: Effect of initial conditions on a circular jet. Exp. Fluids 31, 319-323 (2001)

40. Xu, G., Antonia, R.A.: Effect of different initial conditions on a turbulent round free jet. Exp. Fluids 33, 677-683 (2002)

41. Burattini, P., Antonia, R.A., Rajagopalan, S., Stephens, M.: Effect of initial conditions on the near-field development of a round jet. Exp. Fluids 37, 56-64 (2007)

42. Foysi, H., Mellado, J.P., Sarkar, S.: Large-Eddy simulation of variable-density round and plane jets. Int. J. Heat Fluid Flow 31, 307-314 (2010)

43. Bogey, C., Marsden, O., Bailly, C.: Influence of initial turbulence level on the flow and sound fields of a subsonic jet at a diameter-based reynolds number of $10^{5}$. J. Fluid Mech. 701, 352-385 (2012)

44. Lin, S.P., Woods, D.R.: A branching liquid jet. Phys. Fluids 3, 241-244 (1990) 